\title{
A FREE ENERGY SATISFYING DISCONTINUOUS GALERKIN METHOD FOR ONE-DIMENSIONAL POISSON-NERNST-PLANCK SYSTEMS
}

\author{
HAILIANG LIU $^{\dagger}$ AND ZHONGMING WANG $\ddagger$
}

\begin{abstract}
We design an arbitrary-order free energy satisfying discontinuous Galerkin (DG) method for solving one-dimensional time-dependent Poisson-Nernst-Planck systems. Both the semi-discrete and fully discrete DG methods are shown to satisfy the corresponding discrete free energy dissipation law for positive numerical solutions. Positivities of numerical solutions are enforced by an accuracy-preserving limiter in reference to positive cell averages. Numerical examples are presented to demonstrate the high resolution of the numerical algorithm and to illustrate the proven properties of mass conservation, free energy dissipation, as well as the preservation of steady states.
\end{abstract}

\section{INTRODUCTION}

In this paper, we develop an arbitrary-order free energy satisfying numerical method for solving the initial boundary value problem of the Poisson-Nernst-Planck (PNP) system,

$$
\begin{aligned}
\partial_{t} c_{i} & =\nabla \cdot\left(\nabla c_{i}+q_{i} c_{i} \nabla \psi\right) \quad x \in \Omega, t>0 \\
-\Delta \psi & =\sum_{i=1}^{m} q_{i} c_{i}+\rho_{0}(x), \quad x \in \Omega, t>0, \\
c_{i}(0, x) & =c_{i}^{\text {in }}(x), \quad x \in \Omega, \\
\frac{\partial \psi}{\partial \mathbf{n}} & =\sigma, \quad \frac{\partial c_{i}}{\partial \mathbf{n}}+q_{i} c_{i} \frac{\partial \psi}{\partial \mathbf{n}}=0, \quad x \in \partial \Omega, t>0,
\end{aligned}
$$

where $c_{i}=c_{i}(t, x)$ is the local concentration of $i^{t h}$ charged molecular or ion species with charge $q_{i}$ $(1 \leq i \leq m)$ at the spatial point $x$ and time $t, \Omega \subset \mathbb{R}^{d}$ denotes a connected closed domain with smooth boundary $\partial \Omega, \psi=\psi(t, x)$ is the electrostatic potential governed by the Poisson equation subject to the Neumann boundary data $\sigma$ and the charge density that consists of both fixed charge $\rho_{0}$ and mobile ions, the latter being a linear combination of all the concentrations $c_{i}$. Here $\mathbf{n}$ is the unit outward normal vector on the domain boundary $\partial \Omega$. In this system, the diffusion coefficient, the thermal energy and the dielectric coefficient have been normalized in a dimensionless manner.

The side conditions are necessarily compatible, i.e.,

$$
\int_{\Omega}\left(\sum_{i=1}^{m} q_{i} c_{i}^{\mathrm{in}}(x)+\rho_{0}(x)\right) d x+\int_{\partial \Omega} \sigma d s=0,
$$

for the solvability of the problem.

The PNP system is a mean field approximation of diffusive molecules or ions, and consists of Nernst-Planck (NP) equations that describe the drift and diffusion of ion species, and the Poisson equation that describes the electrostatics interaction. In the process of charge transport

1991 Mathematics Subject Classification. 35K40, 65M60, 65M12, 82C31.

Key words and phrases. Poisson-Nernst-Planck equation, free energy, discontinuous Galerkin methods. 
the fluxes of charge carriers are driven exclusively by processes of diffusion and electric drift. This description using the flux traces back to Nernst [44] and Planck [45], and is accurate for modeling systems with point charges. Applications of this system are found in electrical engineering and electronkinetics [17, 26, 28, 39, 40,42], electrochemistry [23], and biophysics [18, 25].

Three main properties of the solution to (1.1) are the non-negativity, mass conservation and the free energy dissipation, i.e.,

$$
\begin{aligned}
& c_{i}^{\text {in }}(x) \geq 0 \Longrightarrow c_{i}(t, x) \geq 0 \quad \forall t>0, x \in \Omega, \\
& \int_{\Omega} c_{i}(t, x) d x=\int_{\Omega} c_{i}^{\text {in }}(x) d x \quad \forall t>0, \\
& \frac{d}{d t} F=-\sum_{i=1}^{m} \int_{\Omega} c_{i}^{-1}\left|\nabla c_{i}+q_{i} c_{i} \nabla \psi\right|^{2} d x+\int_{\partial \Omega} \partial_{t} \sigma \psi d s,
\end{aligned}
$$

where the free energy $F$ is defined by

$$
F=\int_{\Omega} \sum_{i=1}^{m} c_{i} \log c_{i} d x+\frac{1}{2} \int_{\Omega}\left|\nabla_{x} \psi\right|^{2} d x .
$$

The free energy contains both entropic part and the interaction part: $c_{i} \log c_{i}$ is the entropy related to the Brownian motion of each ion species, and $\frac{1}{2} \int_{\Omega}\left|\nabla_{x} \psi\right|^{2} d x$ is the electrostatic potential of the Coulomb interaction between charged ions. For the PNP system, the density is expected to converge to the equilibrium solution in a closed system (e.g. $\sigma=0$ ) regardless of how initial data are distributed.

These nice mathematical features are crucial for the analytical study of the PNP system. For instance, by some energy estimate with the control of the free energy dissipation, the solution is shown to converge to the thermal equilibrium state as time becomes large, if the boundary conditions are in thermal equilibrium (see, e.g., [22]). Long time behavior was studied in [8], and further in $[1,6]$ with refined convergence rates. Results for the drift-diffusion model regarding existence and asymptotic studies in the case of different boundary conditions may be found in [19-21].

1.1. Existing and proposed methods. Due to the wide variety of devices modeled by the PNP equations, computer simulation for this system of differential equations is of great interest. In addition to being more computationally efficient, PNP models more easily incorporate certain types of boundary conditions that arise in physical systems, such as boundaries of fixed concentration or electrostatic potential. However, the PNP equations present difficulties when computing approximate solutions: it is a strongly coupled system of $m+1$ nonlinear equations, so that computational efficiency plays a critical role in applications of a numerical solver. The transport equations are often convection-dominated, numerical simulation may produce negative ion concentrations or oscillations in the computed solution, if not properly addressed.

In the literature, there are different numerical approximations available for the steady state Poisson-Nernst-Planck equations, see e.g. [2, 9, 26, 39, 42]. Computational algorithms for timedependent PNP systems have also been constructed for both one-dimensional and two or threedimensional models in various chemical/biological applications, and have been combined with the Brownian Dynamics simulations. The proposed algorithms range from finite difference methods $[11-13,27,41,50,51,54]$ to finite element methods $[16,37,38,43,46]$. Many of existing algorithms are introduced to handle specific settings in complex applications, in which one may encounter different numerical obstacles, such as discontinuous coefficients, singular charges, geometric singularities, and nonlinear couplings to accommodate various phenomena exhibited by biological ion channels. 
For a broader overview of proposed algorithms, we refer the interested reader to a recent review article [53].

Given the existing rich literature, the most distinct feature of this work is the use of properties (1.3a)-(1.3c) as a guide to design an arbitrary high order algorithm to efficiently simulate the solution at large times. The most related works to the present one are $[30,31,43]$ and in spirit $[14,15,22,34-36]$. The second order finite difference method introduced in [30] satisfies all three properties in (1.3) at the discrete level. For the class of nonlinear Fokker-Planck (NFP) equations

$$
\partial_{t} c=\nabla_{x} \cdot\left(f(c) \nabla_{x}\left(\psi(x)+H^{\prime}(c)\right)\right),
$$

with the potential $\psi$ given, the high order discontinuous Galerkin method introduced in [31] is shown to satisfy the discrete entropy dissipation law. If the potential $\psi$ is governed by the Poisson equation such as (1.1b), $f(c)=c$ and $H(c)=c \log c$, then (1.5) becomes the PNP system (1.1) with single species. A finite element method to the PNP system is recently introduced in [43] using a logarithmic transformation of the charge carrier densities, while the involved energy estimate resembles the physical energy law that governs the PNP system in the continuous case. The main objective of this work is to develop a high order DG method that incorporates mathematical features (1.3) to handle the coupling of the Poisson equation and the NP system so that the numerical solution remains faithful for long time simulations.

The discontinuous Galerkin (DG) method we present here is a class of finite element methods, using a completely discontinuous piecewise polynomial space for the numerical solution and the test functions. One main advantage of the DG method is the flexibility afforded by local approximation spaces combined with the suitable design of numerical fluxes crossing cell interfaces. More general information about DG methods for elliptic, parabolic, and hyperbolic PDEs can be found in the recent books and lecture notes see,e.g. $[24,47,48]$. The DG discretization we use is motivated by the direct discontinuous Galerkin (DDG) method proposed in $[32,33]$. The main feature in the DDG schemes lies in numerical flux choices for the solution gradient, which involve higher order derivatives evaluated crossing cell interfaces. The entropy satisfying methods recently developed in $[30,31,34-36]$ are the main references for the present work.

The main results in this paper include the formulation of an arbitrary-order DG methods, proofs of the discrete free energy dissipation and mass conservation for both semi-discrete and fully discrete DG methods, and an accuracy-preserving limiter to ensure the positivity preserving property for $c_{i}$. Numerical results are in excellent agreement with the analysis.

We point out that different boundary conditions are used in applications, and they are important in driving the system out of equilibrium and produce the non-vanishing ionic fluxes. The numerical method presented in this work can be easily modified to incorporate different boundary conditions, through selection of appropriate boundary fluxes (see section 2).

1.2. Related models. The PNP system has many variants by simply altering the definition of the charge carrier flux. For example, the PNP system coupled with the Navier-Stokes equation is a basic model in the study of electronkinetics [28]. It reduces to simpler models when some of ion species become trivial or steady. For example, in the case of $m=1$, if $c_{1}=c$ with $q_{1}=1$, we have

$$
\begin{aligned}
& \partial_{t} c=\nabla \cdot(\nabla c+c \nabla \psi) \quad x \in \Omega, t>0, \\
&-\Delta \psi=c+\rho_{0}(x), \quad x \in \Omega, t>0, \\
& c(0, x)=c^{\text {in }}(x), \quad x \in \Omega, \\
& \frac{\partial \psi}{\partial \mathbf{n}}=\sigma, \quad \frac{\partial c}{\partial \mathbf{n}}+c \frac{\partial \psi}{\partial \mathbf{n}}=0, \quad x \in \partial \Omega, t>0 . \\
& 3
\end{aligned}
$$


It may well be the case that when some species still evolve in time, the others are already at the steady states due to different time scales. For example, in the case of $m=2$, if $c_{1}=c$ and $c_{2}=S(x)$ with $q_{1}=1=-q_{2}$, we obtain

$$
\begin{aligned}
\partial_{t} c & =\nabla \cdot(\nabla c+c \nabla \psi) \quad x \in \Omega, t>0, \\
0 & =\nabla \cdot(\nabla S-S \nabla \psi) \quad x \in \Omega, t>0, \\
-\Delta \psi & =c+\rho_{0}(x)+S(x) \quad x \in \Omega, t>0, \\
c(0, x) & =c^{\mathrm{in}}(x), \quad x \in \Omega, \\
\frac{\partial \psi}{\partial \mathbf{n}} & =\sigma, \quad \frac{\partial S}{\partial \mathbf{n}}-\sigma S=0, \quad \frac{\partial c}{\partial \mathbf{n}}+\sigma c=0, \quad x \in \partial \Omega, t>0 .
\end{aligned}
$$

If all ion species are at the equilibrium states $c_{i}=\lambda_{i} e^{-q_{i} \psi}$, with $\lambda_{i}=\int_{\Omega} c^{\text {in }}(x) d x \int_{\Omega} e^{-q_{i} \psi} d x$ so that the total density remains as given initially, the Poisson equation thus becomes a Poisson-Boltzmann equation(PBE) of the form

$$
-\Delta \psi=\sum_{i=1}^{m}\left(q_{i} \int_{\Omega} c_{i}^{\text {in }}(x) d x\right) \frac{e^{-q_{i} \psi}}{\int_{\Omega} e^{-q_{i} \psi} d x}+\rho_{0}(x), \quad x \in \Omega,\left.\quad \frac{\partial \psi}{\partial \mathbf{n}}\right|_{\partial \Omega}=0 .
$$

We should point out that the numerical method presented in this paper may be used as an iterative algorithm to numerically compute the reduced system (1.7) and the nonlocal PBE (1.8), which plays essential roles in chemistry and biophysics.

In a larger context, the concentration equation also links to the general class of aggregation equations with diffusion

$$
\partial_{t} c+\nabla \cdot(c \nabla(G * c))=\Delta c
$$

which has been widely studied in applications such as biological swarms $[4,7,52]$ and chemotaxis $[5,10]$. For chemotaxis, a wide literature exists in relation to the Keller-Segel model (see $[5,10]$ and references therein). The left-hand-side in (1.9) represents the active transport of the density $c$ associated to a non-local velocity field $u=\nabla(G * c)$. The potential $G$ is usually assumed to incorporate attractive interactions among individuals of the group, while repulsive (anti-crowding) interactions are accounted for by the diffusion in the right-hand-side. Of central role in studies of model (1.9), and also particularly relevant to the present research, is the gradient flow formulation of the equation with respect to the free energy

$$
F[c]=\int_{\Omega} c \log c d x-\frac{1}{2} \int_{\Omega} \int_{\Omega} G(x-y) c(x) c(y) d x d y .
$$

1.3. Contents. This paper is organized as follows: in Section 2, we present the DG method for the one dimensional case, and discuss how to deal with different types of boundary conditions; Section 3 is devoted to theoretical analysis for both semi-discrete and fully discrete schemes. We give the details of the numerical algorithm in Section 4, including how to compute the potential $\psi_{h}$, the positivity preserving limiter, and the choice of the ODE solver. Numerical results are

presented in Section 5. Finally, concluding remarks are given in Section 6. Further numerical implementation details are given in the appendix.

\section{DG Discretization IN SPACE}

2.1. The DG scheme. In this section we present a DG scheme for (1.1). We consider a domain $\Omega=[a, b]$ and a mesh, which is not necessarily uniform i.e., a family of $N$ control cells $I_{j}$ such that $I_{j}=\left(x_{j-1 / 2}, x_{j+1 / 2}\right)$ with cell center $x_{j}=\left(x_{j-1 / 2}+x_{j+1 / 2}\right) / 2$. We set

$$
a=x_{1 / 2}<x_{1}<\cdots<x_{N-1 / 2}<x_{N}<x_{N+1 / 2}=b,
$$


and $\Delta x_{j}=x_{j+1 / 2}-x_{j-1 / 2}$.

Define the discontinuous finite element space

$$
V_{h}=\left\{v \in L^{2}(\Omega),\left.\quad v\right|_{I_{j}} \in P^{k}\left(I_{j}\right), j=1, \cdots, N\right\},
$$

where $P^{k}$ denotes polynomials of degree at most $k$. We rewrite the PNP system as follows

$$
\begin{aligned}
\partial_{t} c_{i} & =\partial_{x}\left(c_{i} \partial_{x} p_{i}\right), i=1, \cdots, m, \\
p_{i} & =q_{i} \psi+\log c_{i}, \\
-\partial_{x}^{2} \psi & =\sum_{i=1}^{m} q_{i} c_{i}+\rho_{0}(x),
\end{aligned}
$$

subject to initial data $c_{i}(0, x)=c_{i}^{\text {in }}(x)$, which are necessarily to meet the compatibility requirement:

$$
\int_{\Omega}\left(\sum_{i=1}^{m} q_{i} c_{i}^{\mathrm{in}}(x)+\rho_{0}(x)\right) d x=\int_{\partial \Omega} \sigma d s .
$$

The DG scheme is to find $c_{i h}, p_{i h}, \psi_{h} \in V_{h}$ such that for all $v_{i}, r_{i}, \eta \in V_{h}, i=1, \cdots, m$,

$$
\begin{aligned}
& \int_{I_{j}} \partial_{t} c_{i h} v_{i} d x=-\int_{I_{j}} c_{i h} \partial_{x} p_{i h} \partial_{x} v_{i} d x+\left.\left\{c_{i h}\right\}\left(\widehat{\partial_{x} p_{i h}} v_{i}+\left(p_{i h}-\left\{p_{i h}\right\}\right) \partial_{x} v_{i}\right)\right|_{\partial I_{j}}, \\
& \int_{I_{j}} p_{i h} r_{i} d x=\int_{I_{j}}\left(q_{i} \psi_{h}+\log c_{i h}\right) r_{i} d x, \\
& \int_{I_{j}} \partial_{x} \psi_{h} \partial_{x} \eta d x-\left.\left(\widehat{\partial_{x} \psi_{h}} \eta+\left(\psi_{h}-\left\{\psi_{h}\right\}\right) \partial_{x} \eta\right)\right|_{\partial I_{j}}=\int_{I_{j}}\left(\sum_{i=1}^{m} q_{i} c_{i h}+\rho_{0}\right) \eta d x,
\end{aligned}
$$

where $\widehat{\partial_{x} p_{i h}}=F l\left(p_{i h}\right)$ and $\widehat{\partial_{x} \psi_{h}}=F l\left(\psi_{h}\right)$, with the flux operator $F l$ defined by

$$
F l(w):=\beta_{0} \frac{[w]}{h}+\left\{\partial_{x} w\right\}+\beta_{1} h\left[\partial_{x}^{2} w\right] .
$$

Here we have used the notation: $[q]=q^{+}-q^{-}$, and $\{q\}=\left(q^{+}+q^{-}\right) / 2$, where $q^{+}$and $q^{-}$are the values of $q$ from the right and left cell interfaces, respectively. The parameters $\beta_{0}$ and $\beta_{1}$ in $F l(w)$ are chosen to enforce the free energy satisfying property. The details of how to choose $\beta_{0}$ and $\beta_{1}$ are given and justified in Section 3.

For the ODE system $(2.12)$, we prepare initial data $c_{i h}^{\text {in }}$ by the projection of $c_{i}^{\text {in }}$ on the discontinuous finite element space $V_{h}$ so that

$$
\int_{I_{j}} c_{i h}^{\text {in }}(x) v(x) d x=\int_{I_{j}} c_{i}^{\text {in }}(x) v(x) d x, \quad \forall v \in P^{k}\left(I_{j}\right), \quad i=1, \cdots, m .
$$

2.2. Boundary conditions. The boundary conditions are a critical component of the PNP model and determine important qualitative behavior of the solution. Various boundary conditions may be used with the PNP equations. Here we consider the simplest form of boundary conditions for the concentration and for the electric potential field. For the concentration, one usually ignores any chemical reactions at the solid boundaries and assumes a no flux condition $[41,43,46]$, i.e.,

$$
\frac{\partial c_{i}}{\partial \mathbf{n}}+q_{i} c_{i} \frac{\partial \psi}{\partial \mathbf{n}}=0, \quad x \in \partial \Omega, t>0 .
$$

However, it should be noted that it is possible to account for reactions through, for example, using the Butler-Volmer reaction kinetics [3]. The boundary conditions for the electrostatic potential is however not unique and greatly depends on the problem under investigation. Usually, one ends up either specifying the potential or the charge density on the boundary, which corresponds to 
Dirichlet or Neumann boundary conditions, respectively; or using Robin boundary conditions which model capacitors at the boundary. Any combination of these boundary conditions can be applied to $\psi$. Therefore, at the domain boundary the numerical flux needs to be treated with care so that the pre-specified boundary conditions are properly enforced.

To incorporate the boundary condition (1.1d), we set at both $x_{1 / 2}$ and $x_{N+1 / 2}$,

$$
\begin{aligned}
& F l\left(p_{i h}\right)=0,\left\{p_{i h}\right\}=p_{i h},\left\{c_{i h}\right\}=c_{i h} \quad i=1, \cdots, m, \\
& F l\left(\psi_{h}\right)=\sigma,\left\{\psi_{h}\right\}=\psi_{h} .
\end{aligned}
$$

For other types of boundary conditions, we only need to modify the boundary fluxes accordingly. For instance, for Dirichlet boundary conditions of the form

$$
\psi(t, a)=\psi_{l}, \quad \psi(t, b)=\psi_{r} ; \quad c_{i}(t, a)=c_{i l}, \quad c_{i}(t, b)=c_{i r},
$$

we define $\left\{c_{i h}\right\},\left\{\psi_{i h}\right\}, F l\left(\psi_{i h}\right)$ and $F l\left(p_{i h}\right)$ at the boundary in the following way,

$$
\begin{array}{ll}
\text { at } \quad & x_{1 / 2} \\
& \left\{c_{i h}\right\}=\frac{1}{2}\left(c_{i h}^{+}+c_{i l}\right), \quad\left\{\psi_{h}\right\}=\frac{1}{2}\left(\psi_{l}+\psi_{h}^{+}\right), \\
& F l\left(\psi_{h x}\right)=\beta_{0}\left(\psi_{h}^{+}-\psi_{l}\right) / h+\psi_{h x}^{+}, \\
& F l\left(p_{i h}\right)=-\beta_{0}\left(q_{i} \psi_{l}+\log c_{i l}-p_{i h}^{+}\right) / h+p_{i h x}^{+}, \\
\text {at } \quad & x_{N+1 / 2} \\
& \left\{c_{i h}\right\}=\frac{1}{2}\left(c_{i r}+c_{i h}^{-}\right), \quad\left\{\psi_{h}\right\}=\frac{1}{2}\left(\psi_{r}+\psi_{h}^{-}\right), \\
& F l\left(\psi_{h x}\right)=\beta_{0}\left(\psi_{r}-\psi_{h}^{-}\right) / h+\psi_{h x}^{-}, \\
& F l\left(p_{i h}\right)=\beta_{0}\left(q_{i} \psi_{r}+\log c_{i r}-p_{i h}^{-}\right) / h+p_{i h x}^{-},
\end{array}
$$

where $\beta_{0}$ is the penalty parameter to be chosen.

2.3. Comments on the generalization for multidimensional case. It is straightforward to extend DG formulation (2.12) to multi-dimensional spaces. Let $\Omega$ be a convex, bounded polygonal domain in $\mathbb{R}^{d}(d=2,3)$. We partition $\Omega$ into computational elements denoted by $\mathcal{T}_{h}=\{K\}$, and $h$ denotes the characteristic length of all the elements of $\mathcal{T}_{h}$. As usual, we assume the mesh is conforming and the subdivision is regular. We set the DG finite element space as

$$
V_{h}=\left\{v \in L^{2}(\Omega): \forall K \in \mathcal{T}_{h},\left.v\right|_{K} \in P^{k}(K)\right\},
$$

where $P^{k}(K)$ is the space of polynomial functions of degree at most $k$ on $K$. With such an approximation space, the semi-discrete DG scheme is to find $c_{i h}, p_{i h}, \psi_{h} \in V_{h}$ such that for all $v_{i}, r_{i}, \eta \in V_{h}, i=1, \cdots, m$,

$$
\begin{aligned}
& \int_{K} \partial_{t} c_{i h} v_{i} d x=-\int_{K} c_{i h} \nabla_{x} p_{i h} \cdot \nabla_{x} v_{i} d x+\int_{\partial K}\left\{c_{i h}\right\}\left(\widehat{\partial_{n} p_{i h}} v_{i}+\left(p_{i h}-\left\{p_{i h}\right\}\right) \partial_{n} v_{i}\right) d s, \\
& \int_{K} p_{i h} r_{i} d x=\int_{K}\left(q_{i} \psi_{h}+\log c_{i h}\right) r_{i} d x, \\
& \int_{K} \nabla_{x} \psi_{h} \cdot \nabla_{x} \eta d x-\int_{\partial K}\left(\widehat{\partial_{n} \psi_{h}} \eta+\left(\psi_{h}-\left\{\psi_{h}\right\}\right) \partial_{n} \eta\right) d s=\int_{K}\left(\sum_{i=1}^{m} q_{i} c_{i h}+\rho_{0}\right) \eta d x,
\end{aligned}
$$


where $\widehat{\partial_{n} p_{i h}}=F l_{n}\left(p_{i h}\right)$ and $\widehat{\partial_{n} \psi_{h}}=F l_{n}\left(\psi_{h}\right)$, with the flux operator $F l_{n}$ defined on the interface $e$ by

$$
F l_{n}(w):=\beta_{0} \frac{[w]}{h_{e}}+\left\{\partial_{n} w\right\}+\beta_{1} h_{e}\left[\partial_{n}^{2} w\right] .
$$

Here the normal vector $n$ is assumed to be oriented from $K_{1}$ to $K_{2}$, sharing a common edge (face) $e$, and $h_{e}$ is the characteristic length of $e . \partial_{n} w$ and $\partial_{n}^{2} w$ denote the first and second order derivative along direction $n$, respectively. The average $\{w\}$ and the jump $[w]$ of $w$ on $e$ are as follows:

$$
\{w\}=\frac{1}{2}\left(\left.w\right|_{K_{1}}+\left.w\right|_{K_{2}}\right), \quad[w]=\left.w\right|_{K_{2}}-\left.w\right|_{K_{1}} \quad \forall e \in \partial K_{1} \cap \partial K_{2} .
$$

For $e$ in the set of boundary edges, each numerical solution has a uniquely defined restriction on $e$, while boundary conditions can be weakly enforced through the boundary fluxes in the same way as in the one-dimensional case (see Section 2.2). Most of the one-dimensional analysis presented in this work can be easily carried over to the multi-dimensional case, as long as both the bound $\Gamma\left(\beta_{1}, 1\right)$ as in $(3.24)$, and some mesh-dependent inverse inequalities such as those in Lemma 3.2 can be established. However, for this coupled nonlinear system the implementation of the multidimensional algorithm is much more involved, and will be left in future work.

\section{Properties of the PNP system and the DG Method}

3.1. Properties of the continuous PNP system. Under the zero flux condition for $c_{i}$ in $(1.1 \mathrm{~d})$ and the evolution law (1.1a), the total mass for each $c_{i}(i=1, \cdots, m)$ is conserved in the sense that

$$
\begin{aligned}
\frac{d}{d t} \int_{\Omega} c_{i}(t, x) d x & =\int_{\Omega} \nabla_{x} \cdot\left(\nabla c_{i}+q_{i} c_{i} \nabla \psi\right) d x \\
& =\int_{\partial \Omega}\left(\frac{\partial c_{i}}{\partial \mathbf{n}}+q_{i} c_{i} \frac{\partial \psi}{\partial \mathbf{n}}\right) d s \\
& =0 .
\end{aligned}
$$

Furthermore, assume the Dirichlet boundary condition is given and homogeneous, then the stability of the solution to the PNP system is known $[6,8]$ to be given by the energy law of the form

$$
\frac{d}{d t}\left\{\int_{\Omega} \sum_{i=1}^{m} c_{i}\left(\log c_{i}-1\right)+\frac{1}{2}\left|\nabla_{x} \psi\right|^{2} d x\right\}=-\int_{\Omega} \sum_{i=1}^{m} c_{i}\left|\nabla_{x}\left(\log c_{i}+q_{i} \psi\right)\right|^{2} d x,
$$

where the functional,

$$
\int_{\Omega} \sum_{i=1}^{m} c_{i}\left(\log c_{i}-1\right)+\frac{1}{2}\left|\nabla_{x} \psi\right|^{2} d x
$$

is the energy and

$$
\int_{\Omega} \sum_{i=1}^{m} c_{i}\left|\nabla_{x}\left(\log c_{i}+q_{i} \psi\right)\right|^{2} d x \geq 0
$$

is the rate of dissipation. For the Neumann boundary conditions, we show the corresponding energy law by a formal calculation. First, the free energy (1.4) by the Poisson equation can be rewritten as

$$
F=F_{1}+F_{2}:=\sum_{i=1}^{m} \int_{\Omega} c_{i} \log c_{i} d x+\frac{1}{2} \int_{\Omega}\left|\nabla_{x} \psi\right|^{2} d x
$$


We formally calculate the change rate of each part of the free energy

$$
\begin{aligned}
\frac{d}{d t} F_{1}(t) & =\sum_{i=1}^{m} \int_{\Omega}\left(1+\log c_{i}\right) \partial_{t} c_{i} d x \\
& =-\sum_{i=1}^{m} \int_{\Omega} \nabla_{x}\left(\log c_{i}\right) \cdot\left(\nabla_{x} c_{i}+q_{i} c_{i} \nabla_{x} \psi\right) d x \\
& =-\sum_{i=1}^{m} \int_{\Omega}\left(c_{i}^{-1}\left|\nabla_{x} c_{i}\right|^{2}+q_{i} \nabla_{x} c_{i} \cdot \nabla_{x} \psi\right) d x
\end{aligned}
$$

and

$$
\begin{aligned}
\frac{d}{d t} F_{2}(t) & =\int_{\Omega} \nabla_{x} \psi \cdot \partial_{t}\left(\nabla_{x} \psi\right) d x \\
& =\int_{\partial \Omega} \psi \partial_{t}\left(\partial_{n} \psi\right) d s+\int_{\Omega} \psi \partial_{t}(-\Delta \psi) d x \\
& =\int_{\partial \Omega} \psi \partial_{t} \sigma d s+\sum_{i=1}^{m} \int_{\Omega} \psi\left(q_{i} \partial_{t} c_{i}\right) d x \\
& =\int_{\partial \Omega} \psi \partial_{t} \sigma d s-\sum_{i=1}^{m} \int_{\Omega} \nabla_{x}\left(q_{i} \psi\right) \cdot\left(\nabla_{x} c_{i}+q_{i} c_{i} \nabla_{x} \psi\right) d x \\
& =\int_{\partial \Omega} \psi \partial_{t} \sigma d s-\sum_{i=1}^{m} \int_{\Omega} q_{i} \nabla_{x} \psi \cdot \nabla_{x} c_{i} d x-\sum_{i=1}^{m} \int_{\Omega} q_{i}^{2} c_{i}\left|\nabla_{x} \psi\right|^{2} d x
\end{aligned}
$$

Putting together we obtain

$$
\frac{d}{d t} F=\int_{\partial \Omega} \psi \partial_{t} \sigma d s-\sum_{i=1}^{m} \int_{\Omega} c_{i}^{-1}\left|\nabla_{x} c_{i}+q_{i} c_{i} \nabla_{x} \psi\right|^{2} d x
$$

For the case of $\partial_{t} \sigma=0$ we have

$$
\frac{d}{d t} F=-\sum_{i=1}^{m} \int_{\Omega} c_{i}^{-1}\left|\nabla_{x} c_{i}+q_{i} c_{i} \nabla_{x} \psi\right|^{2} d x \leq 0 .
$$

Remark 3.1. In the special case that $\sigma=0$ and $\rho_{0}=0$, both $F_{1}$ and $F_{2}$ are each decreasing in time, with

$$
\begin{aligned}
& \frac{d}{d t} F_{1}=-\int_{\Omega} \sum_{i=1}^{m} c_{i}^{-1}\left|\nabla_{x} c_{i}\right|^{2} d x-\int_{\Omega}|\Delta \psi|^{2} d x \leq 0, \\
& \frac{d}{d t} F_{2}=-\int_{\Omega}\left(\sum_{i=1}^{m} q_{i}^{2} c_{i}\right)\left|\nabla_{x} \psi\right|^{2} d x-\int_{\Omega}|\Delta \psi|^{2} d x \leq 0 .
\end{aligned}
$$

This can be verified by a direct calculation as

$$
\begin{aligned}
-\sum_{i=1}^{m} \int_{\Omega} q_{i} \nabla_{x} \psi \cdot \nabla_{x} c_{i} d x & =\int_{\Omega}(\Delta \psi) \sum_{i=1}^{m}\left(q_{i} c_{i}\right) d x-\int_{\partial \Omega} \sigma \sum_{i=1}^{m}\left(q_{i} c_{i}\right) d s \\
& =-\int_{\Omega}|\Delta \psi|^{2} d x .
\end{aligned}
$$


3.2. Properties of the semi-discrete DG method. For some $M(x)>0$, we define the weighted bilinear operator

$$
A_{M}(u, v)=\sum_{j=1}^{N} \int_{I_{j}} M \partial_{x} u \partial_{x} v d x+\sum_{j=1}^{N-1}\left(\{M\}\left(\widehat{u_{x}}[v]+\left\{v_{x}\right\}[u]\right)\right)_{j+1 / 2},
$$

and the associated energy norm

$$
\|v\|_{M, E}^{2}=\sum_{j=1}^{N} \int_{I_{j}} M v_{x}^{2} d x+\sum_{j=1}^{N-1} \frac{\beta_{0}}{h}\left(\{M\}[v]^{2}\right)_{j+1 / 2} .
$$

When $M=1$, we denote $\|v\|_{1, E}=\|v\|_{E}$. We also introduce the notation,

$$
\Gamma\left(\beta_{1}, M\right):=\sup _{u \in P^{(k-1)}([-1,1])} \frac{2\left(u(1)-2 \beta_{1} \partial_{\xi} u(1)\right)^{2}}{\int_{-1}^{1} w_{j}(\xi) u^{2}(\xi) d \xi}
$$

with

$$
w_{j}(\xi)=\min \left\{M\left(x_{j}+\frac{h}{2} \xi\right), M\left(x_{j+1}-\frac{h}{2} \xi\right)\right\} .
$$

We recall the following estimates.

Lemma 3.1. (cf. [35]) If $\beta_{0}>\Gamma\left(\beta_{1}, M\right)\{M\}$ at each interface $x_{j+1 / 2}, j=1, \cdots, N-1$, then

$$
A_{M}(v, v) \geq \gamma\|v\|_{M, E}^{2}+\frac{1}{2} \int_{I_{1} \cup I_{N}} M v_{x}^{2} d x
$$

for some $\gamma \in(0,1)$. We also have

$$
\Gamma\left(\beta_{1}, M\right) \geq \frac{2 h\left(\left[\partial_{x} w\right]+\beta_{1} h\left[\partial_{x}^{2}\right]\right)^{2}}{\left(\int_{I_{j}}+\int_{I_{j+1}}\right) M\left(\partial_{x} w\right)^{2} d x} .
$$

Remark 3.2. The additional terms $\frac{1}{2} \int_{I_{1} \cup I_{N}} M v_{x}^{2} d x$ were not used in [35]; here we need them to control boundary contributions.

In the case of $M=1$, the bound $\Gamma\left(\beta_{0}, 1\right)$ is obtained in [29]:

$$
\Gamma\left(\beta_{1}, 1\right)=k^{2}\left(1-\beta_{1}\left(k^{2}-1\right)+\frac{\beta_{1}^{2}}{3}\left(k^{2}-1\right)^{2}\right),
$$

which achieves its minimum $k^{2} / 4$ at $\beta_{1}=\frac{3}{2\left(k^{2}-1\right)}$.

We first examine the existence and uniqueness of $\psi_{h}$ from solving (2.12c). Note that for Neumann boundary data, only $\sigma_{a}$ and $\sigma_{b}$ are pre-specified so that

$$
\sigma_{a}-\sigma_{b}=\int_{a}^{b} \rho(t, x) d x, \quad \rho(t, x)=\rho_{0}(x)+\sum_{i=1}^{m} q_{i} c_{i}(t, x),
$$

yet $\psi(t, a)$ and $\psi(t, b)$ are not given, the exact solution is unique up to an additive constant.

Theorem 3.1. Assume that $\beta_{0}>\Gamma\left(\beta_{1}, 1\right)$, then for each given $\rho$, there exists a unique $\psi_{h}$ to (2.12c) with (2.20) and (2.16), up to an additive constant.

Proof. It suffices to prove the uniqueness since existence is equivalent to uniqueness for this finite dimensional problem. Let $\eta=\psi_{h 1}-\psi_{h 2}$ for two different solutions $\psi_{h i}$, then

$$
A_{1}(\eta, \eta)=0 .
$$


On the other hand, Lemma 3.1 ensures that there exists $\gamma \in(0,1)$ such that

$$
A_{1}(\eta, \eta) \geq \gamma\|\eta\|_{E}^{2}=\gamma\left(\sum_{j=1}^{N} \int_{I_{j}} \eta_{x}^{2} d x+\sum_{j=1}^{N-1} \frac{\beta_{0}}{h}[\eta]_{j+1 / 2}^{2}\right) .
$$

Hence $\eta$ must be a constant.

We next prove the conservation of $c_{i h}$ and the semi-discrete free energy dissipation law.

Theorem 3.2. $\quad$ 1. The semi-discrete scheme is conservative in the sense that each total concentration $c_{i h}(i=1, \cdots, m)$ remains unchanged in time,

$$
\frac{d}{d t} \sum_{j=1}^{N} \int_{I_{j}} c_{i h}(t, x) d x=0, \quad t>0
$$

2. Suppose that $\partial_{t} \sigma=0$ and $c_{i h}(t, x)>0$ in each $I_{j}$, the semi-discrete free energy

$$
F=\sum_{j=1}^{N} \int_{I_{j}}\left[\sum_{i=1}^{m} c_{i h} \log c_{i h}+\frac{1}{2}\left(\sum_{i=1}^{m} q_{i} c_{i h}+\rho_{0}\right) \psi_{h}\right] d x+\frac{1}{2} \int_{\partial \Omega} \sigma \psi_{h} d s
$$

satisfies

$$
\frac{d}{d t} F=-\sum_{i=1}^{m} A_{c_{i h}}\left(p_{i h}, p_{i h}\right)
$$

Moreover,

$$
\frac{d}{d t} F \leq 0
$$

provided $\beta_{0}$ is suitably large, and $\beta_{1}=0$ in $F l\left(\psi_{h}\right)$ defined in (2.20) and (2.16).

Proof. 1. The conservation (3.25) follows from taking $v_{i}=1$ in (2.12a) with summation over $j$.

2. Set

$$
\rho_{h}(t, x)=\sum_{i=1}^{m} q_{i} c_{i h}(t, x)+\rho_{0}(x) .
$$

A direct calculation using $\sum_{j=1}^{N} \int_{I_{j}} \partial_{t} c_{i h} d x=0$ and the assumption of $\sigma_{t}=0$ gives

$$
\begin{aligned}
\frac{d}{d t} F & =\sum_{j=1}^{N} \int_{I_{j}} \sum_{i=1}^{m}\left(1+\ln c_{i h}+q_{i} \psi_{h}\right) \partial_{t} c_{i h} d x+\frac{1}{2} \sum_{j=1}^{N} \int_{I_{j}}\left[\rho_{h} \partial_{t} \psi_{h}-\partial_{t} \rho_{h} \psi_{h}\right] d x+\frac{1}{2} \int_{\partial \Omega} \sigma \partial_{t} \psi_{h} d s \\
& =\sum_{j=1}^{N} \int_{I_{j}} \sum_{i=1}^{m}\left(\ln c_{i h}+q_{i} \psi_{h}\right) \partial_{t} c_{i h} d x+\frac{1}{2} \sum_{j=1}^{N} \int_{I_{j}}\left[\rho_{h} \partial_{t} \psi_{h}-\partial_{t} \rho_{h} \psi_{h}\right] d x+\frac{1}{2} \int_{\partial \Omega} \sigma \partial_{t} \psi_{h} d s .
\end{aligned}
$$

From (2.12b) with $r_{i}=\partial_{t} c_{i h}$ it follows that

$$
\int_{I_{j}}\left(\ln c_{i h}+q_{i} \psi_{h}\right) \partial_{t} c_{i h} d x=\int_{I_{j}} p_{i h} \partial_{t} c_{i h} d x .
$$

Summing (2.12a) in $i, j$ with $v_{i}=p_{i h}$ gives

$$
\sum_{j=1}^{N} \int_{I_{j}} \sum_{i=1}^{m} \partial_{t} c_{i h} p_{i h} d x=-\sum_{j=1}^{N} \int_{I_{j}} \sum_{i=1}^{m} c_{i h}\left|p_{i h x}\right|^{2} d x-\sum_{j=1}^{N-1} \sum_{i=1}^{m}\left(\left\{c_{i h}\right\}\left[p_{i h}\right]\left(\widehat{p_{i h x}}+\left\{p_{i h x}\right\}\right)\right)_{j+1 / 2},
$$


since $\widehat{p}_{h x, \frac{1}{2}}=\widehat{p}_{h x, N+\frac{1}{2}}=0$. Next we sum (2.12c) over all cell index $j$ to obtain

$$
A_{1}\left(\psi_{h}, \eta\right)=\sum_{j=1}^{N} \int_{I_{j}} \rho_{h} \eta d x+\int_{\partial \Omega} \sigma \eta d s .
$$

In one dimensional case,

$$
\int_{\partial \Omega} \sigma \eta d s=\sigma_{b} \eta_{N+1 / 2}^{-}-\sigma_{a} \eta_{1 / 2}^{+}
$$

Taking derivative of (3.27) with respect to $t$ we also have

$$
A_{1}\left(\partial_{t} \psi_{h}, \eta\right)=\sum_{j=1}^{N} \int_{I_{j}} \partial_{t} \rho_{h} \eta d x .
$$

Thus (3.27) with $\eta=\partial_{t} \psi_{h}$ subtracted by (3.28) with $\eta=\psi_{h}$ gives

$$
\begin{aligned}
\sum_{j=1}^{N} \int_{I_{j}}\left[\rho_{h} \partial_{t} \psi_{h}-\partial_{t} \rho_{h} \psi_{h}\right] d x+\int_{\partial \Omega} \sigma \partial_{t} \psi_{h} d s & =A_{1}\left(\psi_{h}, \partial_{t} \psi_{h}\right)-A_{1}\left(\partial_{t} \psi_{h}, \psi_{h}\right) \\
& =\beta_{1} h \sum_{j=1}^{N-1}\left(\left[\partial_{x}^{2} \psi_{h}\right]\left[\partial_{t} \psi_{h}\right]-\left[\partial_{x}^{2} \partial_{t} \psi_{h}\right]\left[\psi_{h}\right]\right)_{j+1 / 2} \\
& =0
\end{aligned}
$$

since $\beta_{1}=0$ in the numerical flux $F l\left(\psi_{h}\right)$ defined in (2.20).

Putting all together we obtain

$$
\begin{aligned}
\frac{d}{d t} F & =-\sum_{j=1}^{N} \int_{I_{j}} \sum_{i=1}^{m} c_{i h}\left|p_{i h x}\right|^{2} d x-\sum_{j=1}^{N-1} \sum_{i=1}^{m}\left(\left\{c_{i h}\right\}\left[p_{i h}\right]\left(\widehat{p_{i h x}}+\left\{p_{i h x}\right\}\right)\right)_{j+1 / 2} \\
& =-\sum_{i=1}^{m} A_{c_{i h}}\left(p_{i h}, p_{i h}\right) \\
& \leq 0
\end{aligned}
$$

if at each interface $\beta_{0}>\max _{i} \Gamma\left(\beta_{1}, c_{i h}\right)\left\{c_{i h}\right\}$, as shown in Lemma 3.1.

Remark 3.3. For $\left(\beta_{0}, \beta_{1}\right)$ in the numerical flux formulation (2.20), we use $\beta_{0}>k^{2}$ and $\beta_{1}=0$ for $\mathrm{Fl}\left(\psi_{h}\right)$. For $\mathrm{Fl}\left(p_{h}\right)$ we need to pick

$$
\beta_{0}>\Gamma\left(\beta_{1}, c_{i h}\right)\left\{c_{i h}\right\}
$$

for numerical solutions $c_{i h}$. For sufficiently small $h$, variation of $c_{i h}$ near each interface is relatively bounded by a factor 2 , hence it suffices to choose

$$
\beta_{0}>2 \Gamma\left(\beta_{1}, 1\right)=2 k^{2}\left(1-\beta_{1}\left(k^{2}-1\right)+\frac{\beta_{1}^{2}}{3}\left(k^{2}-1\right)^{2}\right),
$$

where (3.24) has been used. For $k>1$, one choice is $\beta_{1}=\frac{3}{2\left(k^{2}-1\right)}$ so that $\beta_{0}>2 \Gamma\left(\beta_{1}, 1\right)=k^{2} / 2$. Therefore it suffices to take $\beta_{0}>k^{2}$ in (2.20) for both $F l\left(\psi_{h}\right)$ and $F l\left(p_{h}\right)$. The choices of $\left(\beta_{0}, \beta_{1}\right)$ in our numerical simulation all fall within the range $\beta_{0}>k^{2}$. 
3.3. Properties of the fully discrete DG method. In order to preserve the free energy dissipation law at each time step, the time step restriction is needed when using an explicit time discretization. We now discuss this issue by taking the Euler first order time discretization of (2.12) with uniform time step $\Delta t$ : find $c_{i h}^{n+1}, \psi_{h}^{n+1} \in V_{h}$ such that for any $v_{i}, r_{i}, \eta \in V_{h}$,

$$
\begin{aligned}
& \int_{I_{j}} D_{t} c_{i h}^{n} v_{i} d x=-\int_{I_{j}} c_{i h}^{n} \partial_{x} p_{i h}^{n} \partial_{x} v_{i} d x+\left.\left\{c_{i h}^{n}\right\}\left(\widehat{\partial_{x} p_{i h}^{n}} v_{i}+\left(p_{i h}^{n}-\left\{p_{i h}^{n}\right\}\right) \partial_{x} v_{i}\right)\right|_{\partial I_{j}}, \\
& \int_{I_{j}} p_{i h}^{n} r_{i} d x=\int_{I_{j}}\left(q_{i} \psi_{h}^{n}+\log c_{i h}^{n}\right) r_{i} d x \\
& \int_{I_{j}} \partial_{x} \psi_{h}^{n} \partial_{x} \eta d x-\left.\left(\widehat{\partial_{x} \psi_{h}^{n}} \eta+\left(\psi_{h}^{n}-\left\{\psi_{h}^{n}\right\}\right) \partial_{x} \eta\right)\right|_{\partial I_{j}}=\int_{I_{j}}\left[\sum_{i=1}^{m} q_{i} c_{i h}^{n}+\rho_{0}\right] \eta d x .
\end{aligned}
$$

Here and in what follows, we use the notation for any function $w^{n}(x)$ as

$$
D_{t} w^{n}=\frac{w^{n+1}-w^{n}}{\Delta t}
$$

We recall some basic estimates:

Lemma 3.2. (cf. [31, Lemma 3.2]) The following inequalities hold for any $v \in V_{h}$ :

$$
\begin{aligned}
& \sum_{j=1}^{N} \int_{I_{j}} v_{x}^{2} d x \leq \frac{k(k+1)^{2}(k+2)}{h^{2}} \sum_{j=1}^{N} \int_{I_{j}} v^{2} d x, \\
& \sum_{j=1}^{N-1}[v]_{j+1 / 2}^{2} \leq \frac{4(k+1)^{2}}{h} \sum_{j=1}^{N} \int_{I_{j}} v^{2} d x, \\
& \sum_{j=1}^{N-1}\left\{v_{x}\right\}_{j+1 / 2}^{2} \leq \frac{k^{3}(k+1)^{2}(k+2)}{h^{2}} \sum_{j=1}^{N} \int_{I_{j}} v^{2} d x .
\end{aligned}
$$

Based on these estimates we show several properties of the fully discretized scheme.

Theorem 3.3. $\quad$ 1. The fully discrete scheme (3.29) is conservative in the sense that each total concentration $c_{i h}^{n}(x)(i=1, \cdots, m)$ remains unchanged in time,

$$
\sum_{j=1}^{N} \int_{I_{j}} c_{i h}^{n} d x=\sum_{j=1}^{N} \int_{I_{j}} c_{i h}^{n+1} d x, \quad i=1, \cdots, m, \quad t>0 .
$$

2. Assume that $c_{i h}^{n}(x)>0$ in each $I_{j}$. There exists $\mu^{*}>0$ such that if the mesh ratio $\mu=\frac{\Delta t}{\Delta x^{2}} \in\left(0, \mu^{*}\right)$, then the fully discrete free energy

$$
F^{n}=\sum_{j=1}^{N} \int_{I_{j}}\left[\sum_{i=1}^{m} c_{i h}^{n} \log c_{i h}^{n}+\frac{1}{2}\left(\sum_{i=1}^{m} q_{i} c_{i h}^{n}+\rho_{0}\right) \psi_{h}^{n}\right] d x+\frac{1}{2} \int_{\partial \Omega} \sigma \psi_{h}^{n} d s
$$

satisfies

$$
D_{t} F^{n} \leq-\frac{1}{2} \sum_{i=1}^{m} A_{c_{i h}^{n}}\left(p_{i h}^{n}, p_{i h}^{n}\right)
$$

Moreover,

$$
F^{n+1} \leq F^{n}
$$

provided that $\beta_{0}$ is suitably large, and $\beta_{1}=0$ in $F l\left(\psi_{h}\right)$ defined in (2.20) and (2.16). 
Proof. Set

$$
\rho_{h}^{n}=\sum_{i=1}^{m} q_{i} c_{i h}^{n}+\rho_{0}
$$

and sum over $j$ in (3.29) to obtain

$$
\begin{aligned}
& \int_{\Omega} D_{t} c_{i h}^{n} v_{i} d x=-A_{c_{i h}^{n}}\left(p_{i h}^{n}, v_{i}\right), \\
& \int_{\Omega} p_{i h}^{n} r_{i} d x=\int_{\Omega}\left(q_{i} \psi_{h}^{n}+\log c_{i h}^{n}\right) r_{i} d x \\
& A_{1}\left(\psi_{h}^{n}, \eta\right)=\int_{\Omega} \rho_{h}^{n} \eta d x+\int_{\partial \Omega} \sigma \eta d s .
\end{aligned}
$$

1. Taking $v_{i}=1$ in (3.34a) yields (3.31).

2. Taking $v_{i}=p_{i h}^{n}$ in (3.34a), and $r_{i}=D_{t} c_{i h}^{n}$ in (3.34b), respectively, and summing over $m$, we obtain

$$
\int_{\Omega} \sum_{i=1}^{m}\left(D_{t} c_{i h}^{n}\right) p_{i h}^{n} d x=-\sum_{i=1}^{m} A_{c_{i h}^{n}}\left(p_{i h}^{n}, p_{i h}^{n}\right)
$$

and

$$
\int_{\Omega} \sum_{i=1}^{m} p_{i h}^{n}\left(D_{t} c_{i h}^{n}\right) d x=\int_{\Omega} \sum_{i=1}^{m}\left(q_{i} \psi_{h}^{n}+\log c_{i h}^{n}\right) D_{t} c_{i h}^{n} d x .
$$

From $(3.34 \mathrm{c})$ we see that

$$
A_{1}\left(\psi_{h}^{n+1}-\psi_{h}^{n}, \eta\right)=\int_{\Omega}\left(\rho_{h}^{n+1}-\rho_{h}^{n}\right) \eta d x
$$

This with $\eta=\psi_{h}^{n}$ and $\rho_{h}^{n}=\sum_{i=1}^{m} q_{i} c_{i h}^{n}+\rho_{0}(x)$ yields

$$
\int_{\Omega} \sum_{i=1}^{m} q_{i} \psi_{h}^{n}\left(c_{i h}^{n+1}-c_{i h}^{n}\right) d x=\int_{\Omega}\left(\rho_{h}^{n+1}-\rho_{h}^{n}\right) \psi_{h}^{n} d x=A_{1}\left(\psi_{h}^{n+1}-\psi_{h}^{n}, \psi_{h}^{n}\right) .
$$

These relations lead to

$$
\int_{\Omega} \sum_{i=1}^{m} \log c_{i h}^{n}\left(c_{i h}^{n+1}-c_{i h}^{n}\right) d x=-\Delta t \sum_{i=1}^{m} A_{c_{i h}^{n}}\left(p_{i h}^{n}-p_{i h}^{n}\right)-A_{1}\left(\psi_{h}^{n+1}-\psi_{h}^{n}, \psi_{h}^{n}\right) .
$$

Note that (3.34c) with $\eta=\psi_{h}^{n+1}-\psi_{h}^{n}$ subtracted by (3.35) with $\eta=\psi_{h}^{n}$ gives

$$
\begin{gathered}
\int_{\Omega}\left[\rho_{h}^{n}\left(\psi_{h}^{n+1}-\psi_{h}^{n}\right)-\left(\rho_{h}^{n+1}-\rho^{n}\right) \psi_{h}^{n}\right] d x+\int_{\partial \Omega} \sigma\left(\psi_{h}^{n+1}-\psi_{h}^{n}\right) d s \\
=A_{1}\left(\psi_{h}^{n}, \psi_{h}^{n+1}-\psi_{h}^{n}\right)-A_{1}\left(\psi_{h}^{n+1}-\psi_{h}^{n}, \psi_{h}^{n}\right)=0 .
\end{gathered}
$$

Also taking $\eta=\psi_{h}^{n+1}+\psi_{h}^{n}$ in (3.35) we obtain

$$
\int_{\Omega}\left(\rho_{h}^{n+1}-\rho_{h}^{n}\right)\left(\psi_{h}^{n+1}+\psi_{h}^{n}\right) d x=A_{1}\left(\psi_{h}^{n+1}-\psi_{h}^{n}, \psi_{h}^{n+1}+\psi_{h}^{n}\right) .
$$

Adding these up leads to

$$
\int_{\partial \Omega} \sigma\left(\psi_{h}^{n+1}-\psi_{h}^{n}\right) d s+\int_{\Omega}\left(\rho_{h}^{n+1} \psi_{h}^{n+1}-\rho_{h}^{n} \psi_{h}^{n}\right) d x=A_{1}\left(\psi_{h}^{n+1}-\psi_{h}^{n}, \psi_{h}^{n+1}+\psi_{h}^{n}\right) .
$$


With (3.37) and (3.38) we proceed to evaluate

$$
\begin{aligned}
F^{n+1}-F^{n}= & \sum_{i=1}^{m} \int_{\Omega}\left[c_{i h}^{n+1} \log c_{i h}^{n+1}-c_{i h}^{n} \log c_{i h}^{n}\right] d x+\frac{1}{2} \int_{\partial \Omega} \sigma\left(\psi_{h}^{n+1}-\psi_{h}^{n}\right) d s \\
& +\frac{1}{2} \int_{\Omega}\left(\rho_{h}^{n+1} \psi_{h}^{n+1}-\rho_{h}^{n} \psi_{h}^{n}\right) d x \\
= & \sum_{i=1}^{m} \int_{\Omega} \log c_{i h}^{n}\left(c_{i h}^{n+1}-c_{i h}^{n}\right) d x+\sum_{i=1}^{m} \int_{\Omega} c_{i h}^{n+1} \log \left(\frac{c_{i h}^{n+1}}{c_{i h}^{n}}\right) d x \\
& \quad+\frac{1}{2} A_{1}\left(\psi_{h}^{n+1}-\psi_{h}^{n}, \psi_{h}^{n+1}+\psi_{h}^{n}\right) \\
= & -\Delta t \sum_{i=1}^{m} A_{c_{i h}^{n}}\left(p_{i h}^{n}, p_{i h}^{n}\right)+\sum_{i=1}^{m} \int_{\Omega} c_{i h}^{n+1} \log \left(\frac{c_{i h}^{n+1}}{c_{i h}^{n}}\right) d x+\frac{1}{2} A_{1}\left(\psi_{h}^{n+1}-\psi_{h}^{n}, \psi_{h}^{n+1}-\psi_{h}^{n}\right) \\
= & -\Delta t \sum_{i=1}^{m} A_{c_{i h}^{n}}\left(p_{i h}^{n}, p_{i h}^{n}\right)+\sum_{i=1}^{m} \int_{\Omega} c_{i h}^{n} \log \left(\frac{c_{i h}^{n+1}}{c_{i h}^{n}}\right) d x+G^{n} \\
\leq & -\Delta t \sum_{i=1}^{m} A_{c_{i h}^{n}}\left(p_{i h}^{n}, p_{i h}^{n}\right)+G^{n},
\end{aligned}
$$

where the non-positivity of the second term is based on $\log X \leq X-1$ and the conservation of $\sum_{i=1}^{m} \int_{\Omega} c_{i h}^{n} d x$. Here

$$
G^{n}=\sum_{i=1}^{m} \int_{\Omega}\left(c_{i h}^{n+1}-c_{i h}^{n}\right) \log \left(\frac{c_{i h}^{n+1}}{c_{i h}^{n}}\right) d x+\frac{1}{2} A_{1}\left(\psi_{h}^{n+1}-\psi_{h}^{n}, \psi_{h}^{n+1}-\psi_{h}^{n}\right)=: G_{1}^{n}+G_{2}^{n},
$$

which is non-negative, yet small.

It remains to figure out a sufficient restriction on the mesh ratio $\mu=\Delta t /(\Delta x)^{2}$ so that

$$
G^{n} \leq \frac{\Delta t}{2} \sum_{i=1}^{m} A_{c_{i h}^{n}}\left(p_{i h}^{n}, p_{i h}^{n}\right)
$$

In (3.34a), we take $v_{i}=D_{t} c_{i h}^{n}$ and use the Young inequality $a b \leq \frac{1}{4 \epsilon} a^{2}+\epsilon b^{2}$ to obtain

$$
\begin{aligned}
\sum_{j=1}^{N} \int_{I_{j}} v_{i}^{2} d x & =-\sum_{j=1}^{N} \int_{I_{j}} c_{i h}^{n} \partial_{x} p_{i h}^{n} \partial_{x} v_{i} d x-\left.\sum_{j=1}^{N-1}\left\{c_{i h}^{n}\right\}\left(\widehat{\partial_{x} p_{i h}^{n}}\left[v_{i}\right]+\left\{\partial_{x} v_{i}\right\}\left[p_{i h}^{n}\right]\right)\right|_{x_{j+\frac{1}{2}}} \\
& \leq \frac{1}{4 \epsilon_{1} h^{2}} \sum_{j=1}^{N} \int_{I_{j}}\left(c_{i h}^{n}\right)^{2}\left|\partial_{x} p_{i h}^{n}\right|^{2} d x+\epsilon_{1} h^{2} \sum_{j=1}^{N} \int_{I_{j}}\left|\partial_{x} v_{i}\right|^{2} d x \\
& +\left.\frac{1}{4 \epsilon_{2} h} \sum_{j=1}^{N-1}\left\{c_{i h}^{n}\right\}^{2}\left|\widehat{\partial_{x} p_{i h}^{n}}\right|^{2}\right|_{x_{j+\frac{1}{2}}}+\left.\epsilon_{2} h \sum_{j=1}^{N-1}\left[v_{i}\right]^{2}\right|_{x_{j+\frac{1}{2}}} \\
& +\left.\frac{1}{4 \epsilon_{3} h^{3}} \sum_{j=1}^{N-1}\left\{c_{i h}^{n}\right\}^{2}\left[p_{i h}^{n}\right]^{2}\right|_{x_{j+\frac{1}{2}}}+\left.\epsilon_{3} h^{3} \sum_{j=1}^{N-1}\left\{\partial_{x} v_{i}\right\}^{2}\right|_{x_{j+\frac{1}{2}}} .
\end{aligned}
$$


The use of inequalities (3.30) in Lemma 3.2 leads to

$$
\begin{aligned}
& \epsilon_{1} h^{2} \sum_{j=1}^{N} \int_{I_{j}}\left|\partial_{x} v_{i}\right|^{2} d x+\left.\epsilon_{2} h \sum_{j=1}^{N-1}\left[v_{i}\right]^{2}\right|_{x_{j+\frac{1}{2}}}+\left.\epsilon_{3} h^{3} \sum_{j=1}^{N-1}\left[\partial_{x} v_{i}\right]^{2}\right|_{x_{j+\frac{1}{2}}} \\
& \leq(k+1)^{2}\left(k(k+2) \epsilon_{1}+4 \epsilon_{2}+k^{3}(k+2) \epsilon_{3}\right) \sum_{j=1}^{N} \int_{I_{j}} v_{i}^{2} d x \\
& =\frac{3}{4} \sum_{j=1}^{N} \int_{I_{j}} v_{i}^{2} d x,
\end{aligned}
$$

if we choose $\epsilon_{i}$ as

$$
\left(4 \epsilon_{1}\right)^{-1}=k(k+1)^{2}(k+2),\left(4 \epsilon_{2}\right)^{-1}=4(k+1)^{2}, \quad\left(4 \epsilon_{3}\right)^{-1}=k^{3}(k+1)^{2}(k+2) .
$$

This gives

$$
\begin{aligned}
& \frac{1}{4} \sum_{j=1}^{N} \int_{I_{j}} v_{i}^{2} d x \leq \frac{k(k+1)^{2}(k+2)}{h^{2}} \sum_{j=1}^{N} \int_{I_{j}}\left(c_{i h}^{n}\right)^{2}\left|\partial_{x} p_{i h}^{n}\right|^{2} d x \\
& \quad+\left.\frac{k^{3}(k+1)^{2}(k+2)}{h^{3}} \sum_{j=1}^{N-1}\left\{\left(c_{i h}^{n}\right)\right\}^{2}\left[p_{i h}^{n}\right]^{2}\right|_{x_{j+\frac{1}{2}}}+\left.\frac{4(k+1)^{2}}{h} \sum_{j=1}^{N-1}\left\{c_{i h}^{n}\right\}^{2}\left|\widehat{\partial_{x} p_{i h}^{n}}\right|^{2}\right|_{x_{j+\frac{1}{2}}} .
\end{aligned}
$$

It is clear that the first two terms are bounded by $h^{-2}\left\|c_{i h}^{n}(\cdot)\right\|_{\infty}\left\|p_{i h}^{n}\right\|_{c_{i h}^{n}, E}^{2}$. We next show that the last term is also bounded by $h^{-2}\left\|c_{i h}^{n}(\cdot)\right\|_{\infty}\left\|p_{i h}^{n}\right\|_{c_{i h}^{n}, E}^{2}$, up to constant multiplication factors. Note that

$$
\begin{aligned}
\left.\left|\widehat{\partial_{x} p_{i h}^{n}}\right|^{2}\right|_{x_{j+\frac{1}{2}}} & =\left|\left\{\partial_{x} p_{i h}^{n}\right\}+\beta_{0} \frac{\left[p_{i h}^{n}\right]}{h}+\beta_{1} h\left[\partial_{x}^{2} p_{i h}^{n}\right]\right|^{2} \\
& \leq 2\left(\beta_{0}^{2} \frac{\left[p_{i h}^{n}\right]^{2}}{h^{2}}+\left(\left\{\partial_{x} p_{i h}^{n}\right\}+\beta_{1} h\left[\partial_{x}^{2} p_{i h}^{n}\right]\right)^{2}\right) .
\end{aligned}
$$

From (3.23) it follows that

$$
\left.\left(\left\{\partial_{x} p_{i h}^{n}\right\}+\beta_{1} h\left[\partial_{x}^{2} p_{i h}^{n}\right]\right)^{2}\right|_{x_{j+\frac{1}{2}}} \leq \frac{\Gamma\left(\beta_{1}, c_{i h}^{n}\right)}{2 h}\left(\int_{I_{j}}+\int_{I_{j+1}}\right) c_{i h}^{n}\left|\partial_{x} p_{i h}\right|^{2} d x .
$$

Hence

$$
\begin{aligned}
\left.\sum_{j=1}^{N-1}\left\{c_{i h}^{n}\right\}^{2}\left|\widehat{\partial_{x} p_{i h}^{n}}\right|^{2}\right|_{x_{j+\frac{1}{2}}} & \leq \frac{2}{h} \max \left\{\beta_{0}, \Gamma\left(\beta_{1}, c_{i h}^{n}\right)\left\{c_{i h}^{n}\right\}\right\}\left\|c_{i h}^{n}(\cdot)\right\|_{\infty}\left\|p_{i h}^{n}\right\|_{c_{i h}^{n}, E}^{2} \\
& =\frac{2 \beta_{0}}{h}\left\|c_{i h}^{n}(\cdot)\right\|_{\infty}\left\|p_{i h}^{n}\right\|_{c_{i h}^{n}, E}^{2} .
\end{aligned}
$$

Upon insertion into (3.40) we obtain

$$
\sum_{j=1}^{N} \int_{I_{j}} v_{i}^{2} d x \leq \frac{C\left(k, \beta_{0}\right)\left\|c_{i h}^{n}(\cdot)\right\| \|_{\infty}}{h^{2}}\left\|p_{i h}^{n}\right\|_{c_{i h}^{n}, E}^{2},
$$

where

$$
\left.C\left(k, \beta_{0}\right):=4(k+1)^{2}\left(k(k+2) \max \left\{1, k^{2} / \beta_{0}\right\}+8 \beta_{0}\right\}\right) .
$$


Note that

$$
\begin{aligned}
G_{1}^{n} & \leq \sum_{i=1}^{m} \int_{\Omega} \frac{\left(c_{i h}^{n+1}-c_{i h}^{n}\right)^{2}}{c_{i h}^{n}} d x=\sum_{i=1}^{m} \int_{\Omega} \frac{v_{i}^{2}}{c_{i h}^{n}} d x(\Delta t)^{2} \\
& \leq \mu \Delta t \frac{C\left(k, \beta_{0}\right)\left\|c_{i h}^{n}(\cdot)\right\|_{\infty}}{\min _{i, x} c_{i h}^{n}} \sum_{i=1}^{m}\left\|p_{i h}^{n}\right\|_{c_{i h}^{n}, E}^{2} \\
& \leq \mu \Delta t \frac{C\left(k, \beta_{0}\right)\left\|c_{i h}^{n}(\cdot)\right\|_{\infty}}{\gamma \min _{i, x} c_{i h}^{n}} \sum_{i=1}^{m} A_{c_{i h}^{n}}\left(p_{i h}^{n}, p_{i h}^{n}\right) \\
& \leq \frac{\Delta t}{4} \sum_{i=1}^{m} A_{c_{i h}^{n}}\left(p_{i h}^{n}, p_{i h}^{n}\right),
\end{aligned}
$$

if the mesh ratio satisfies

$$
\mu \leq \frac{\gamma \min _{i, x} c_{i h}^{n}}{4 C\left(k, \beta_{0}\right) \max _{i}\left\|c_{i h}^{n}(\cdot)\right\|_{\infty}} .
$$

It remains to bound $G_{2}^{n}$. From (3.35) it follows that for $\xi:=\psi_{h}^{n+1}-\psi_{h}^{n}$,

$$
A_{1}(\xi, \xi)=\sum_{i=1}^{m} q_{i} \int_{\Omega}\left(c_{i h}^{n+1}-c_{i h}^{n}\right) \xi d x=\Delta t \sum_{i=1}^{m} q_{i} \int_{\Omega} v_{i} \xi d x \leq \Delta t \sum_{i=1}^{m}\left|q_{i}\right|\left\|v_{i}\right\|\|\xi\| .
$$

Note that $A_{1}(\cdot, \cdot)$ is a symmetric bilinear operator ( $A_{1}(\xi, \xi)=0$ if and only if $\xi \equiv$ const, therefore

$$
c=\inf _{\left\{\xi \in V_{h}, \xi \neq \text { const }\right\}} \frac{h^{2} A_{1}(\xi, \xi)}{\|\xi\|^{2}},
$$

is positive. A simple rescaling suggests that $c$ is also independent of $h$. Hence $\|\xi\|^{2} \leq c^{-1} h^{2} A_{1}(\xi, \xi)$, which when inserted into $(3.42)$, set $C_{1}=\sum_{i=1}^{m} q_{i}^{2} / c^{2}$, leads to

$$
\begin{aligned}
A_{1}(\xi, \xi) & \leq C_{1}(\Delta t)^{2} h^{2} \sum_{i=1}^{m}\left\|v_{i}\right\|^{2} \leq C_{1} C\left(k, \beta_{0}\right) \max _{i}\left\|c_{i h}^{n}(\cdot)\right\|_{\infty}(\Delta t)^{2} \sum_{i=1}^{m}\left\|p_{i h}^{n}\right\|_{c_{i h}^{n}, E}^{2} \\
& \leq C_{1}(\Delta t)^{2} \frac{C\left(k, \beta_{0}\right) \max _{i}\left\|c_{i h}^{n}(\cdot)\right\|_{\infty}}{\gamma} \sum_{i=1}^{m} A_{c_{i h}^{n}}\left(p_{i h}^{n}, p_{i h}^{n}\right) \\
& \leq \frac{\Delta t}{4} \sum_{i=1}^{m} A_{c_{i h}^{n}}\left(p_{i h}^{n}, p_{i h}^{n}\right),
\end{aligned}
$$

as long as the time step also satisfies

$$
\Delta t \leq \frac{\gamma}{4 C_{1} C\left(k, \beta_{0}\right) \max _{i}\left\|c_{i h}^{n}(\cdot)\right\|_{\infty}} .
$$

Collecting the above estimates on $G_{i}^{n}(i=1,2)$ we obtain $(3.39)$, if we take

$$
\mu^{*}=\frac{\gamma}{4 C\left(k, \beta_{0}\right) \max _{i}\left\|c_{i h}^{n}(\cdot)\right\|_{\infty}} \min \left\{C_{1}^{-1} h^{-2}, \min _{i, x} c_{i h}^{n}\right\} .
$$

This ends the proof.

Remark 3.4. In Theorem 3.3, $c_{i h}(x)$ is assumed to be positive in each cell $I_{j}$ to make $p_{i h}=$ $q_{i} \psi_{h}+\log c_{i h}$ well defined. In numerical simulations, we enforce this by imposing a limiter defined in (4.50), based on positive cell averages. As shown in [31], such a limiter does not destroy the order of accuracy. Moreover, positivity of cell averages for each $c_{i h}$ is achieved for $\beta_{0}>1$ and 
$\beta_{1} \in(1 / 8,1 / 4)$ when the coupling potential $\psi$ is zero. For the general PNP system, we numerically identify parameter pairs $\left(\beta_{0}, \beta_{1}\right)$ so that cell averages remain positive, as confirmed in Example 1.

In our numerical simulation with $k=1,2,3$, we use the second order explicit Runge-Kutta method (RK2, also called Heun's method) for time discretization to solve the ODE system of the form $\dot{a}=\mathfrak{L}(\mathbf{a}, \mathbf{t})$ :

$$
\begin{aligned}
\mathbf{a}^{(1)} & =\mathbf{a}^{n}+\Delta t \mathfrak{L}\left(\mathbf{a}^{n}, t_{n}\right), \\
\mathbf{a}^{*} & =\mathbf{a}^{(1)}+\Delta t \mathfrak{L}\left(\mathbf{a}^{1}, t_{n+1}\right), \\
\mathbf{a}^{n+1} & =\frac{1}{2} \mathbf{a}^{n}+\frac{1}{2} \mathbf{a}^{*} .
\end{aligned}
$$

Corollary 3.1. Consider the RK2 time discretization(3.43). Assume that $c_{i h}^{n}(x)$ and the intermediate states $c_{i h}^{(1)}$ and $c_{i h}^{*}$ are all positive in each $I_{j}$. There exists $\mu^{*}>0$ such that if the mesh ratio $\mu=\frac{\Delta t}{\Delta x^{2}} \in\left(0, \mu^{*}\right)$, then the fully discrete free energy

$$
F\left(c_{h}^{n}, \psi_{h}^{n}\right)=\sum_{j=1}^{N} \int_{I_{j}}\left[\sum_{i=1}^{m} c_{i h}^{n} \log c_{i h}^{n}+\frac{1}{2}\left(\sum_{i=1}^{m} q_{i} c_{i h}^{n}+\rho_{0}\right) \psi_{h}^{n}\right] d x+\frac{1}{2} \int_{\partial \Omega} \sigma \psi_{h}^{n} d s
$$

satisfies

$$
F^{n+1} \leq F^{n}
$$

provided that $\beta_{0}$ is suitably large, and $\beta_{1}=0$ in $F l\left(\psi_{h}\right)$ defined in (2.20) and (2.16).

Proof. From (3.33) in Theorem 3.3 it follows that

$$
F^{*}:=F\left(c_{h}^{*}, \psi_{h}^{*}\right) \leq F^{(1)}:=F\left(c_{h}^{(1)}, \psi_{h}^{(1)}\right) \leq F^{n}:=F\left(c_{h}^{n}, \psi_{h}^{n}\right) .
$$

Note from (3.34), $F^{n}$ can also be written as

$$
F^{n}=\sum_{j=1}^{N} \int_{I_{j}} \sum_{i=1}^{m} c_{i h}^{n} \log \left(c_{i h}^{n}\right) d x+\frac{1}{2} A_{1}\left(\psi_{h}^{n}, \psi_{h}^{n}\right) .
$$

Hence

$$
F^{n+1}=\sum_{j=1}^{N} \int_{I_{j}}\left[\sum_{i=1}^{m}\left(\frac{1}{2} c_{i h}^{n}+\frac{1}{2} c_{i h}^{*}\right) \log \left(\frac{1}{2} c_{i h}^{n}+\frac{1}{2} c_{i h}^{*}\right)\right] d x+\frac{1}{2} A_{1}\left(\frac{1}{2} \psi_{h}^{n}+\frac{1}{2} \psi_{h}^{*}, \frac{1}{2} \psi_{h}^{n}+\frac{1}{2} \psi_{h}^{*}\right),
$$

where by (3.43), we have used

$$
c_{i h}^{n+1}=\frac{1}{2} c_{i h}^{n}+\frac{1}{2} c_{i h}^{*} ; \quad \psi_{h}^{n+1}=\frac{1}{2} \psi_{h}^{n}+\frac{1}{2} \psi_{h}^{*} .
$$

Using the fact that $A_{1}(w, w)$ is a convex functional in $w$ in the sense that

$$
A_{1}(\theta u+(1-\theta) v, \theta u+(1-\theta) v) \leq \theta A_{1}(u, u)+(1-\theta) A_{1}(v, v), \forall \theta \in[0,1],
$$

which may be verified by the following identity

$$
\theta A_{1}(u, u)+(1-\theta) A_{1}(v, v)-A_{1}(\theta u+(1-\theta) v, \theta u+(1-\theta) v)=\theta(1-\theta) A_{1}(u-v, u-v)
$$

and $A_{1}(u-v, u-v) \geq 0$ from (3.22). Thus

$$
A_{1}\left(\frac{1}{2} \psi_{h}^{n}+\frac{1}{2} \psi_{h}^{*}, \frac{1}{2} \psi_{h}^{n}+\frac{1}{2} \psi_{h}^{*}\right) \leq \frac{1}{2} A_{1}\left(\psi^{n}, \psi^{n}\right)+\frac{1}{2} A_{1}\left(\psi^{*}, \psi^{*}\right) .
$$


Since $c \log c$ is convex in $c$, we also have

$$
\left(\frac{1}{2} c_{i h}^{n}+\frac{1}{2} c_{i h}^{*}\right) \log \left(\frac{1}{2} c_{i h}^{n}+\frac{1}{2} c_{i h}^{*}\right) \leq \frac{1}{2} c_{i h}^{n} \log \left(c_{i h}^{n}\right)+\frac{1}{2} c_{i h}^{*} \log \left(c_{i h}^{*}\right)
$$

Equations (3.45) and (3.46) imply that

$$
F^{n+1} \leq \frac{1}{2} F^{n}+\frac{1}{2} F^{*}
$$

This together with (3.44) leads to $F^{n+1} \leq F^{n}$, as claimed.

Remark 3.5. Corollary 3.1 suggests that the DG scheme (2.12) with the time evolution by the strong stability preserving (SSP) RungeKutta method [49] does not increase the free energy at each time step, as long as the time step is suitably small. Hence high order SSP Runge-Kutta time discretization can be used for high order DG simulations, e.g., $k \geq 4$.

Remark 3.6. The time step restriction $\Delta t \sim O(\Delta x)^{2}$ is obviously a drawback of the explicit time discretization. Usually one would use implicit in time discretization for diffusion and explicit time discretization for the nonlinear drift term (called IMEX in the literature) so that the time step restriction could be relaxed. Unfortunately, formulation (2.11) does not support such a separation.

3.4. Preservation of steady states. If we start with initial data $c_{i h}^{0}$, already at steady states, i.e., $\log c_{i h}^{0}+q_{i} \psi_{h}^{0}(x)=C_{i}$, it follows from (3.29b) that $p_{i h}^{0}=C_{i}$. Furthermore, (3.29a) implies that $c_{i h}^{1}=c_{i h}^{0} \in V_{h}$, which when inserted into (3.29c) gives $\psi_{h}^{1}=\psi_{h}^{0}$ (up to an constant, fixed to 0); hence $\log c_{i h}^{1}+q_{i} \psi_{h}^{1}(x)=C_{i}$. By induction we have

$$
\log c_{i h}^{n}+q_{i} \psi_{h}^{n}(x)=C_{i} \quad \forall n \in \mathbb{N} .
$$

This says that the DG scheme (3.29) preserves steady states. Moreover, we can show that in some cases the numerical solution tends asymptotically toward a steady state, independent of initial data. More precisely, we have the following result.

Theorem 3.4. Let the assumptions in Theorem 3.3 be met, and $\left(c_{i h}^{n}, p_{i h}^{n}, \psi_{h}^{n}\right)$ be the numerical solution to the fully discrete $D G$ scheme (3.29), then the limits of $\left(c_{i h}^{n}, p_{i h}^{n}, \psi_{h}^{n}\right)$ as $n \rightarrow \infty$ satisfy

$$
p_{i h}^{*}=C_{i}, \quad \log c_{i h}^{n}+q_{i} \psi_{h}^{n}(x) \in C_{i}+V_{h}^{\perp},
$$

where $C_{i}$ are some constants.

Proof. Since $F^{n}$ is non-increasing and bounded from below, we have

$$
\lim _{n \rightarrow \infty} F^{n}=\inf \left\{F^{n}\right\} .
$$

Observe from (3.32) that

$$
F^{n+1}-F^{n} \leq-\frac{\Delta t}{2} \sum_{i=1}^{m} A_{c_{i h}^{n}}\left(p_{i h}^{n}, p_{i h}^{n}\right) \leq 0 .
$$

When passing to the limit $n \rightarrow \infty$ we have $\lim _{n \rightarrow \infty} \sum_{i=1}^{m} A_{c_{i h}^{n}}\left(p_{i h}^{n}, p_{i h}^{n}\right)=0$. This and the coercivity of $A_{c_{i h}^{n}}\left(p_{i h}^{n}, p_{i h}^{n}\right)$ imply the limit of $p_{i h}^{n}$, denoted by $p_{i h}^{*}$, must be constant in each computational cell and the whole domain. These when inserted into $(3.29 \mathrm{~b})$ gives the desired result. The proof is complete. 


\section{Numerical Implementation}

4.1. Computing $\psi_{h}$. In order to compute a unique $\psi_{h}$, we fix $\psi(a)$ as being given in one dimensional setting, and define

$$
\begin{aligned}
& F l\left(\psi_{h}\right)(a)=\beta_{0} \frac{\left(\psi_{h}^{+}-\psi(a)\right)}{h}+\frac{1}{2}\left(\sigma_{a}+\psi_{h x}^{+}\right), \quad\{\psi\}=\left(\psi_{h}^{+}+\psi(a)\right) / 2, \\
& F l\left(\psi_{h}\right)(b)=\sigma_{b}, \quad\{\psi\}=\psi_{h}^{-} .
\end{aligned}
$$

We add (2.12c) over all $j=1 \cdots N$ and use the modified boundary condition (4.47) to obtain the following

$$
A\left(\psi_{h}, v\right)=L(v), \quad \forall v \in V_{h},
$$

where

$$
A\left(\psi_{h}, v\right)=A_{1}\left(\psi_{h}, v\right)+\left[\left(\beta_{0} \frac{\psi_{h}^{+}}{h}+\frac{1}{2} \psi_{h x}\right) v^{+}+\frac{1}{2} \psi_{h}^{+} v_{x}^{+}\right]_{x_{1 / 2}}
$$

and

$$
L(v)=\int_{a}^{b} \rho(x) v(x) d x+\left[\left(\beta_{0} \frac{\psi(a)}{h}-\frac{1}{2} \sigma_{a}\right) v^{+}+\frac{1}{2} \psi(a) v_{x}^{+}\right]_{x_{1 / 2}}+\sigma_{b} v_{N+1 / 2}^{-} .
$$

Lemma 4.1. For $\beta_{0} \geq \max \left\{\Gamma\left(\beta_{1}, 1\right), k^{2}\right\}$, and $\rho$ given, there exists a unique $\psi_{h}$ to (2.12c) with (2.20) and the boundary fluxes in (4.47).

Proof. For the same reason as mentioned earlier, it suffices to prove the uniqueness. Let $v=$ $\psi_{h 1}-\psi_{h 2}$ for two different solutions $\psi_{h i}, i=1,2$, then

$$
A(v, v)=0 .
$$

Note that

$$
\begin{aligned}
A(v, v) & =A_{1}(v, v)+\frac{\beta_{0}}{h}\left(v_{1 / 2}^{+}\right)^{2}+\left(v v_{x}\right)_{1 / 2}^{+} \\
& \geq \gamma\|v\|_{E}^{2}+\frac{1}{2} \int_{I_{1}} v_{x}^{2} d x+\frac{\beta_{0}}{2 h}\left(v_{1 / 2}^{+}\right)^{2}-\frac{h}{2 \beta_{0}}\left(v_{x}^{+}\right)_{1 / 2}^{2} \\
& \geq \gamma\|v\|_{E}^{2}+\frac{\beta_{0}}{2 h}\left(v_{1 / 2}^{+}\right)^{2},
\end{aligned}
$$

provided that $\beta_{0}$ is large enough so that

$$
\beta_{0} \geq \sup _{v \in P^{k}\left(I_{1}\right)} \frac{h\left(v_{x}\right)_{1 / 2}^{2}}{\int_{I_{1}} v_{x}^{2} d x}=\sup _{\eta \in P^{k-1}([-1,1])} \frac{2 u^{2}(1)}{\int_{-1}^{1} u^{2}(\xi) d \xi}=\Gamma(0,1)=k^{2} .
$$

Hence every term on the right of (4.49) must be zero, which yields $v \equiv 0$. Uniqueness thus follows.

Remark 4.1. The above result provides a guide for the choices of $\left(\beta_{0}, \beta_{1}\right)$ in numerically solving the Poisson equation. We shall take $\beta_{1}=0$ in solving the Poisson equation so that to also ensure the entropy dissipation property (see Theorem 3.2), hence it suffices to take $\beta_{0}>k^{2}=\Gamma(0,1)$. 
4.2. Positivity-preserving limiter. In the scheme formulation involving the projection of $p_{i}=$ $q_{i} \psi+\log c_{i}$, chemical concentrations $c_{i h}$ need to be strictly positive at each time step, and we follow [31] to enforce positivity through some accuracy-preserving limiter based on positive cell averages.

Let $w_{h} \in P^{k}\left(I_{j}\right)$ be an approximation to a smooth function $w(x) \geq 0$, with cell averages $\bar{w}_{j}>\delta$ for $\delta$ being some small positive parameter or zero. We then consider another polynomial in $P^{k}\left(I_{j}\right)$ so that

$$
w_{h}^{\delta}(x)=\bar{w}_{j}+\frac{\bar{w}_{j}-\delta}{\bar{w}_{j}-\min _{I_{j}} w_{h}(x)}\left(w_{h}(x)-\bar{w}_{j}\right), \quad \text { if } \min _{I_{j}} w_{h}(x)<\delta .
$$

This reconstruction maintains same cell averages and satisfies

$$
\min _{I_{j}} w^{\delta}(x) \geq \delta
$$

Lemma 4.2 (cf. [31]). If $\bar{w}_{j}>\delta$, then $w^{\delta}$ satisfies the estimate

$$
\left|w^{\delta}(x)-w_{h}(x)\right| \leq C(k)\left(\left\|w_{h}(x)-w(x)\right\|_{\infty}+\delta\right), \quad \forall x \in I_{j},
$$

where $C(k)$ is a constant depending on $k$. This says that the reconstructed $w^{\delta}(x)$ in $(4.50)$ does not destroy the accuracy when $\delta<h^{k+1}$.

4.3. Algorithm. The algorithm can be summarized in following steps.

1. (Initialization) Project $c_{i}^{\text {in }}(x)$ onto $V_{h}$, as formulated in (2.14), to obtain $c_{i h}^{0}(x)$.

2. (Reconstruction) From $c_{i h}^{n}(x)$, apply, if necessary, the reconstruction (4.50) to $c_{i h}^{n}$ to ensure that in each cell $c_{i h}^{n}>\delta$.

3. (Poisson solver) Solve (2.12c) to obtain $\psi_{h}^{n}$ subject to the modified boundary fluxes (4.47).

4. (Projection) Solve $(2.12 \mathrm{~b})$ to obtain $p_{i h}^{n}$.

5. (Update) Solve (2.12a) to update $c_{i h}^{n+1}$ with some Runge-Kutta (RK) ODE solver.

6. Repeat steps $2-5$ until final time $T$.

The implementation details are deferred to Appendix A.

\section{Numerical Examples}

In this section, we present a selected set of examples in order to numerically validate our DDG scheme. In $\S 5.1$, we construct an example with exact solution known, and examine the order of accuracy by numerical convergence tests, while we quantify $l_{1}$ errors defined by

$$
\left\|u_{h}-u_{r e f}\right\|_{l_{1}}=\sum_{j=1}^{N} \int_{I_{j}}\left|u_{h}(x)-u_{r e f}(x)\right| d x,
$$

with the integral on $I_{j}$ evaluated by a 4 -point Gaussian quadrature method and $u_{r e f}$ being the exact solution. Long time simulation is also performed to illustrate how the positivity of cell averages propagates when using proper choices of $\left(\beta_{0}, \beta_{1}\right)$. $\$ 5.2$ is devoted to demonstrate the mass conservation, energy dissipation and preservation of the steady state. In $\S 5.3$ and $\S 5.4$, we apply the DDG scheme to a non-monovalent system and a reduced single species system. 
5.1. Cell average and convergence test. In $\Omega=[0,1]$, we consider

$$
\begin{aligned}
\partial_{t} c_{1} & =\partial_{x}\left(\partial_{x} c_{1}+q_{1} c_{1} \partial_{x} \psi\right)+f_{1}, \\
\partial_{t} c_{2} & =\partial_{x}\left(\partial_{x} c_{2}+q_{2} c_{2} \partial_{x} \psi\right)+f_{2}, \\
-\partial_{x}^{2} \psi & =q_{1} c_{1}+q_{2} c_{2}, \\
\partial_{x} \psi(t, 0) & =0, \quad \partial_{x} \psi(t, 1)=-e^{-t} / 60, \\
\partial_{x} c_{i} & +q_{i} c_{i} \partial_{x} \psi=0, \quad x=0,1,
\end{aligned}
$$

with

$$
\begin{aligned}
& f_{1}=\frac{\left(50 x^{9}-198 x^{8}+292 x^{7}-189 x^{6}+45 x^{5}\right)}{30 e^{2 t}}+\frac{\left(-x^{4}+2 x^{3}-13 x^{2}+12 x-2\right)}{e^{t}}, \\
& f_{2}=\frac{(x-1)\left(110 x^{9}-430 x^{8}+623 x^{7}-393 x^{6}+90 x^{5}\right)}{60 e^{2 t}}+\frac{(x-1)\left(x^{4}-2 x^{3}+21 x^{2}-16 x+2\right)}{e^{t}} .
\end{aligned}
$$

This system, with $q_{1}=1$ and $q_{2}=-1$, admits exact solutions

$$
\begin{aligned}
& c_{1}=x^{2}(1-x)^{2} e^{-t}, \\
& c_{2}=x^{2}(1-x)^{3} e^{-t} \\
& \psi=-\left(10 x^{7}-28 x^{6}+21 x^{5}\right) e^{-t} / 420 .
\end{aligned}
$$

We also set $\psi(t, 0)=0$ to pick out a particular solution since $\psi$ is unique up to an additive constant. This extra condition is numerically enforced according to (4.47).

We first test positivity of cell averages for $P^{2}$ polynomials with $\left(\beta_{0}, \beta_{1}\right)=(4,1 / 12)$ in $(2.20)$ for $p_{i h}$. Note that the reconstruction is necessary in this example since $c_{i}=0<\delta$ at $x=0,1$. Our simulation with the reconstruction (4.50) up to $T=100$ indicates that the cell averages remain positive.

Table 5.1 displays both $l_{1}$ errors and orders of convergence when using $P^{k}$ elements at $T=0.1$. We observe that order of convergence is roughly of $k+1$. Figure 5.1 shows the numerical solution at different times. In the top of Figure 5.1, we observe that the numerical solutions (dots) match the exact solutions (solid line) at $t=1$ very well. At $t=5$, our numerical approximation still captures the solution profile very well when the magnitude of the concentrations is close to zero $\left(\sim 10^{-4}\right)$. In the presence of the source terms $f_{1}$ and $f_{2}$, one should not expect to have either mass conservation or free energy decay.

TABLE 5.1. Error table of Example 1 at $T=0.1$

\begin{tabular}{|c|l|c|c|c|c|c|c|}
\hline$\left(k, \beta_{0}, \beta_{1}\right)$ & $\mathrm{h}$ & $c_{1}$ error & order & $c_{2}$ error & order & $\psi$ error & order \\
\hline & 0.2 & 0.023279 & - & 0.031295 & - & 0.0033241 & - \\
$(1,2,-)$ & 0.1 & 0.0037603 & 2.5043 & 0.0059588 & 2.2582 & 0.0009351 & 1.8578 \\
& 0.05 & 0.00065589 & 2.4414 & 0.0012548 & 2.1909 & 0.0002603 & 1.8718 \\
& 0.025 & 0.00012745 & 2.3635 & 0.00028581 & 2.1343 & $6.9808 \mathrm{e}-05$ & 1.8987 \\
\hline \multirow{3}{*}{$(2,4,1 / 12)$} & 0.2 & 0.0028937 & - & 0.0030675 & - & 0.0012417 & - \\
& 0.1 & 0.00018926 & 3.6436 & 0.00024835 & 3.4352 & 0.00010034 & 3.4636 \\
& 0.05 & $1.391 \mathrm{e}-05$ & 3.4981 & $2.2705 \mathrm{e}-05$ & 3.3395 & $9.1444 \mathrm{e}-06$ & 3.3808 \\
& 0.025 & $1.4824 \mathrm{e}-06$ & 3.2301 & $2.4238 \mathrm{e}-06$ & 3.2277 & $9.2476 \mathrm{e}-07$ & 3.3057 \\
\hline \multirow{3}{*}{$(3,15,1 / 4)$} & 0.2 & 0.0030963 & - & 0.0029231 & - & 0.0011002 & - \\
& 0.1 & 0.00023282 & 4.0764 & 0.00021924 & 3.9254 & $7.4195 \mathrm{e}-05$ & 4.3897 \\
& 0.05 & $1.7512 \mathrm{e}-05$ & 4.2480 & $1.6857 \mathrm{e}-05$ & 4.0196 & $5.4161 \mathrm{e}-06$ & 4.6393 \\
& 0.025 & $6.4483 \mathrm{e}-07$ & 4.7633 & $8.3344 \mathrm{e}-07$ & 4.3381 & $1.1946 \mathrm{e}-07$ & 5.5027 \\
\hline
\end{tabular}


FiguRE 5.1. Numerical solution versus exact solution at $t=1$ and $t=5$
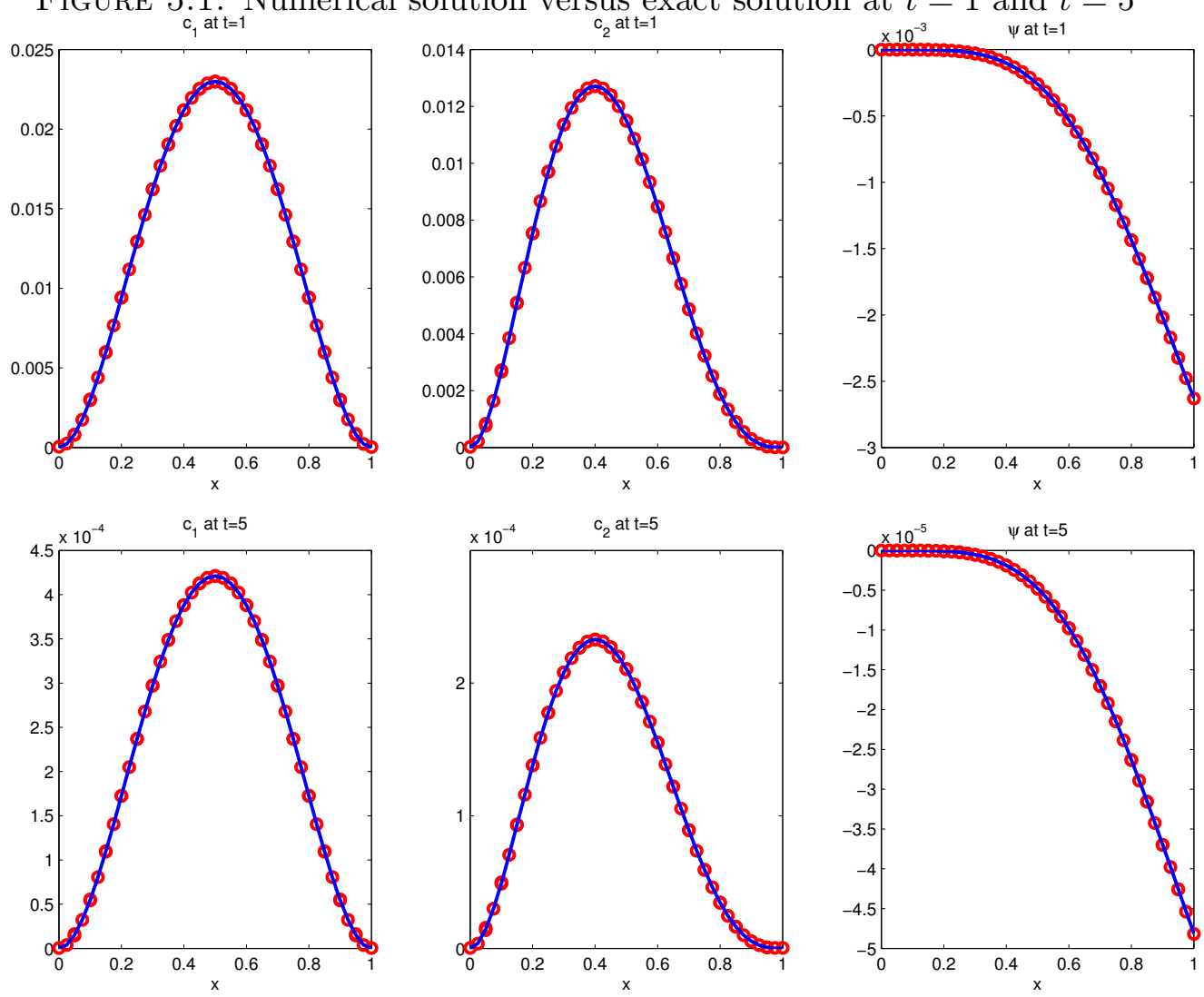

Solid line: exact solutions; dots: numerical solutions

5.2. Mass conservation and free energy dissipation. We consider the following problem on the domain $[0,1]$,

$$
\begin{gathered}
\partial_{t} c_{i}=\partial_{x}\left(\partial_{x} c_{i}+q_{i} c_{i} \partial_{x} \psi\right), \quad i=1,2 \\
-\partial_{x}^{2} \psi=q_{1} c_{1}+q_{2} c_{2}, \\
\partial_{x} \psi=0, \quad \partial_{x} c_{i}=0, \quad i=1,2, \quad x=0,1,
\end{gathered}
$$

where $q_{1}$ and $q_{2}$ are set to be 1 and -1 , respectively, with initial conditions

$$
c_{1}^{\text {in }}(x)=1+\pi \sin (\pi x), \quad c_{2}^{\text {in }}(x)=4-2 x,
$$

which satisfies the compatibility condition (1.2).

With zero flux for concentration $c_{1}$ and $c_{2}$, this model example is to test the conservation of total mass and free energy decay. In Figure 5.2 (top), we see the snapshots of $c_{1}, c_{2}$ and $\psi$ at $t=0,0.01,0.1,0.8,1$. We observe that the solutions at $t=0.8$ and $t=1$ are indistinguishable. Obviously the solution is converging to the steady state, which has constant $c_{1}=3, c_{2}=3$ and $\psi=0$. Figure 5.2 (bottom) shows the energy decay (see the change on the right vertical axis) and conservation of mass (see the left vertical axis). We see that the total mass of $c_{1}$ and $c_{2}$ stays constant all the time while the free energy is decreasing monotonically. In fact the free energy levels off after $t=0.2$, at which the system is already in steady state. 
Figure 5.2. Temporal evolution of the solutions
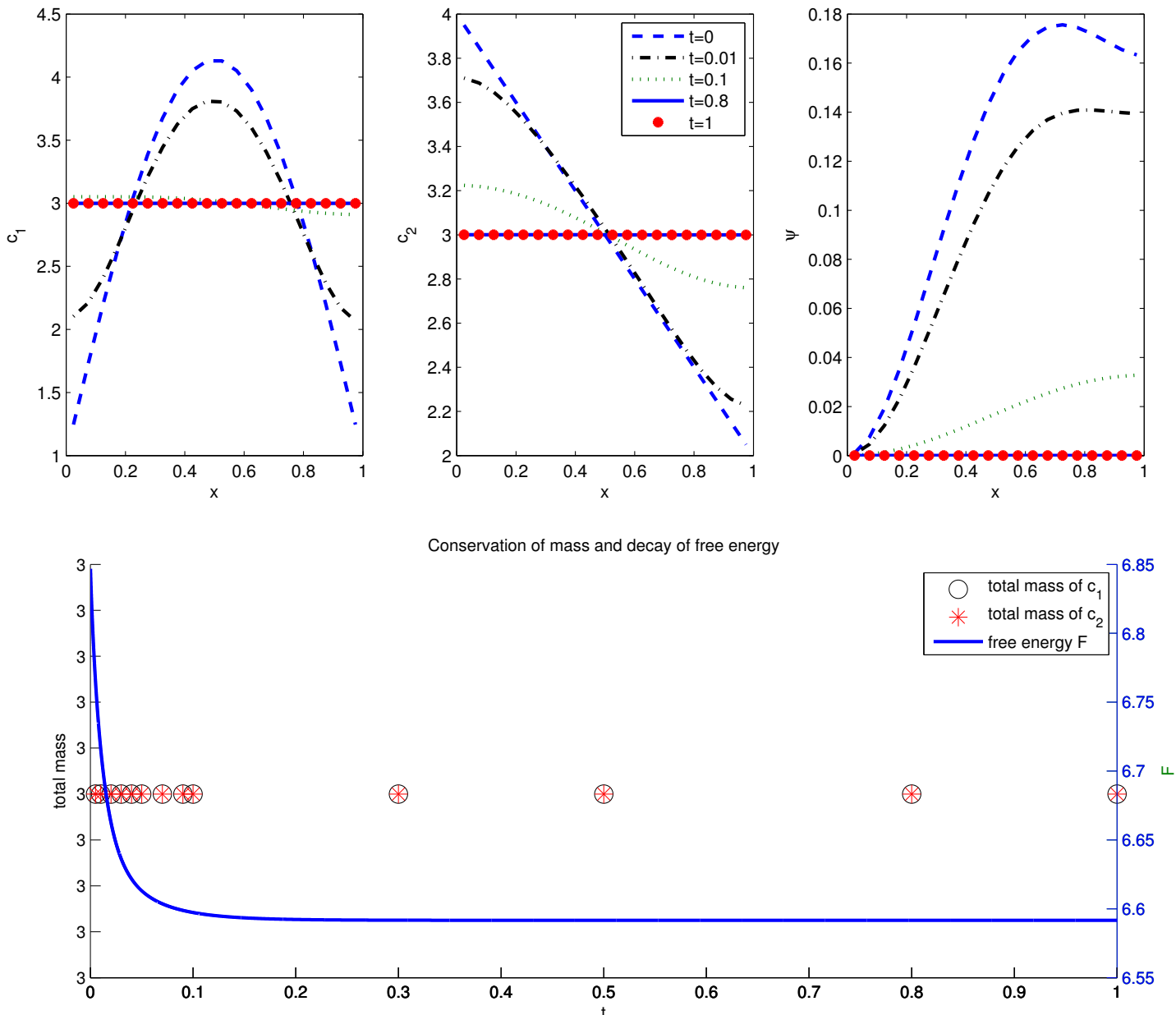

5.3. Non-monovalent system and nonzero fixed charge. We consider the following nonmonovalent system (monovalent if $q_{1}=-q_{2}=1$ ) with nonzero fixed charge $\rho_{0}$ in $[0,1]$.

$$
\begin{aligned}
\partial_{t} c_{1} & =\partial_{x}\left(\partial_{x} c_{1}+q_{1} c_{1} \psi_{x}\right), \\
\partial_{t} c_{2} & =\partial_{x}\left(\partial_{x} c_{2}+q_{2} c_{2} \psi_{x}\right), \\
-\partial_{x}^{2} \psi & =q_{1} c_{1}+q_{2} c_{2}+\rho_{0},
\end{aligned}
$$

with $q_{1}=1, q_{2}=-2$ and $\rho_{0}=12(x-0.5)^{2}$. The initial and boundary conditions are

$$
\begin{gathered}
c_{1}^{\text {in }}(x)=2+12(x-0.5)^{2}, \quad c_{2}^{\text {in }}(x)=1+2 x, \\
\partial_{x} c_{i}+q_{i} c_{i} \partial_{x} \psi=0, \quad x=0,1, \\
\partial_{x} \psi(t, 0)=\partial_{x} \psi(t, 1)=0,
\end{gathered}
$$

where the compatibility condition (1.2) is satisfied since $\int_{0}^{1}\left(q_{1} c_{1}^{\text {in }}+q_{2} c_{2}^{\text {in }}+\rho_{0}\right) d x=0$.

In Figure 5.3 (top) are the snapshots of $c_{1}, c_{2}$ and $\psi$ at $t=0,0.01,0.1,0.8,1$. Figure 5.3 (bottom) shows the energy decay (see the change on the right vertical axis) and conservation of mass (see the left vertical axis). The concentrations $c_{1}$ and $c_{2}$ have different total mass but both are conserved 
in time. We observe that the system is at steady states after $t=0.2$, which are no longer constants due to the nonzero fixed charge $\rho_{0}$.

FiguRE 5.3. Non-monovalent system and nonzero fixed charge
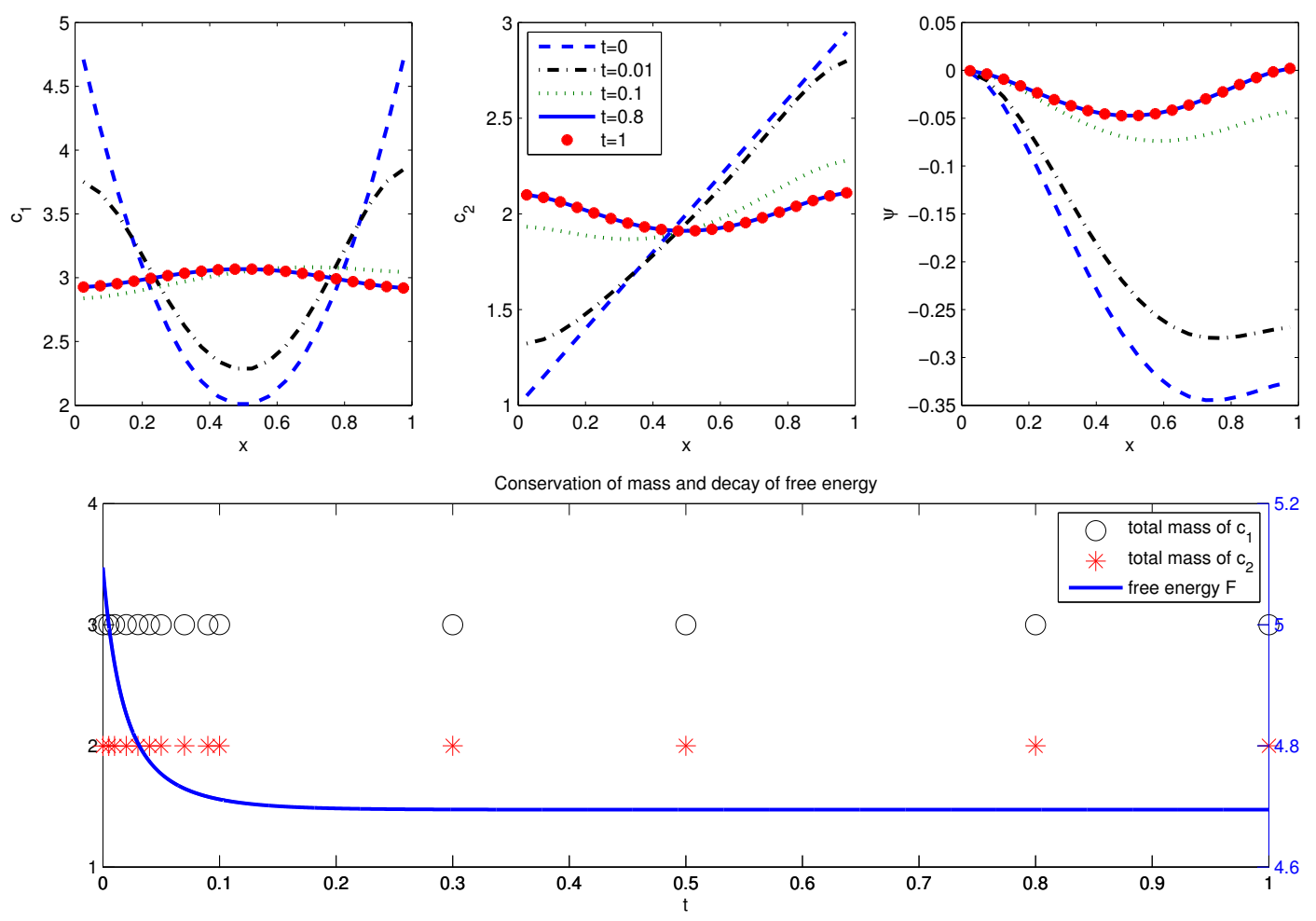

5.4. Single species. Finally we consider the reduced model (1.6) of single species. The problem is

$$
\begin{aligned}
\partial_{t} c & =\partial_{x}\left(\partial_{x} c+c \partial_{x} \psi\right), \quad x \in[0,1], t>0, \\
-\partial_{x}^{2} \psi & =c, \quad x \in[0,1],
\end{aligned}
$$

subject to initial and boundary conditions

$$
c(0, x)=2-x ; \quad \partial_{x} \psi(t, 0)=0 ; \quad \partial_{x} \psi(t, 1)=-3 / 2 ; \quad \partial_{x} c+c \partial_{x} \psi=0, \quad x=0,1,
$$

which satisfies the compatibility condition (1.2).

Figure 5.4 displays the dynamic behavior of $c$ (top left) and of $\psi$ (top right), as well as the mass conservation and energy decay (bottom). Note that the steady state of the concentration $c$ is not a constant in this case due to the nonzero boundary flux $\partial_{x} \psi(t, 1)$, i.e., ions are attracted to the boundary $x=1$.

\section{Concluding REMARKS}

In this paper, we developed an arbitrary high order DG method to solve initial boundary value problems for the Poisson-Nernst-Planck system, which is a mean field type model for concentrations of chemical species. The semi-discrete DG method has been shown to conserve the mass and satisfy the corresponding discrete free energy dissipation law. The fully discrete DG method with the 
FiguRE 5.4. Single species
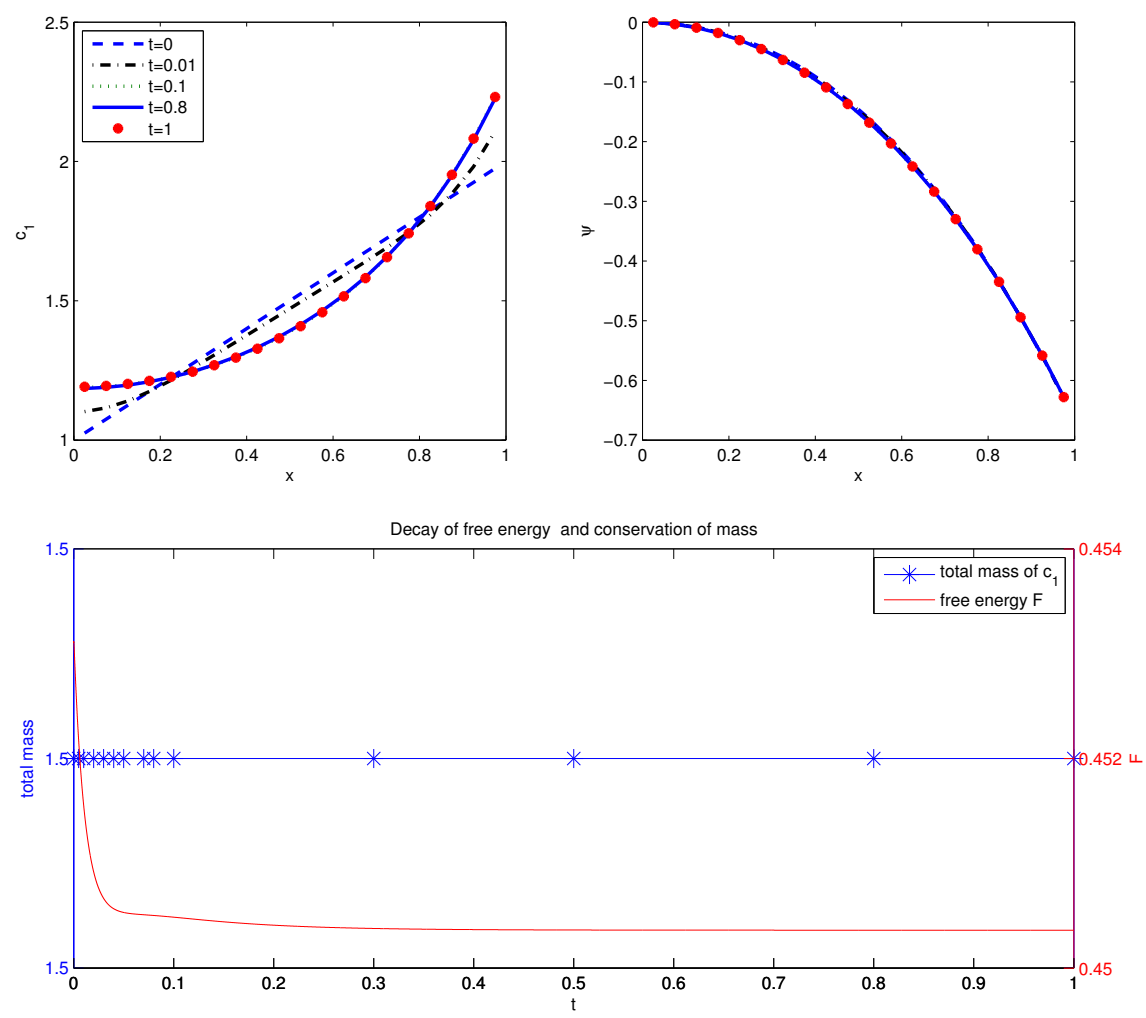

Euler forward time discretization also satisfies the free energy dissipation law under a restriction on the time step relative to the square of the spatial mesh size. Moreover, the free energy does not increase at each time step for the RK2 method (3.43) as well as for the strong stability preserving (SSP) RungeKutta method of any high order. The method also preserves the steady states. These nice properties are also confirmed by our numerical tests. For proper choices of numerical flux parameters $\left(\beta_{0}, \beta_{1}\right)$ in our numerical experiments, we find that each cell average remains positive if it is initially positive. This is needed to update numerical solutions at each time step so they are positive. In future work we will apply our method to multi-dimensional problems and explore more efficient ways to preserve positivity of numerical solutions.

\section{ACKNOWLEDGMEnTs}

This work was supported by the National Science Foundation under Grant DMS1312636 and by NSF Grant RNMS (Ki-Net) 1107291.

\section{Appendix A}

In this appendix, we give details related to the implementation of the method.

The $k$ th order basis functions in a 1-D standard reference element $\xi \in[-1,1]$ are taken as the Legendre polynomials $\left\{L_{l}(\xi)\right\}_{l=0}^{k}$. The numerical solutions $c_{i j}(t, x), p_{i j}(t, x)$ and $\psi_{i}(t, x)$ in each 
cell $I_{j}$, after dropping subscript $h$ for convenience, can be expressed as

$$
\begin{aligned}
& c_{i j}(t, x)=\sum_{l=0}^{k} c_{i j}^{l}(t) L_{l}(\xi)=L^{\top}(\xi) c_{i j}(t), \\
& \psi_{j}(t, x)=\sum_{l=0}^{k} \psi_{j}^{l}(t) L_{l}(\xi)=L^{\top}(\xi) \psi_{j}(t), \\
& p_{i j}(t, x)=\sum_{l=0}^{k} p_{i j}^{l}(t) L_{l}(\xi)=L^{\top}(\xi) p_{i j}(t),
\end{aligned}
$$

using the map $x=x_{j}+\frac{h}{2} \xi$, with notation $L^{\top}=\left(L_{0}, L_{1}, \cdots, L_{k}\right)$ and $c_{i j}=\left(c_{i j}^{0}, \cdots, c_{i j}^{k}\right)^{\top}$. With such an expression, the numerical fluxes at $x_{j+1 / 2}$ become

$$
\begin{aligned}
h \widehat{\partial_{x} p_{i}}= & \beta_{0}\left(L^{\top}(-1) p_{i, j+1}-L^{\top}(1) p_{i j}\right)+\left(L_{\xi}^{\top}(-1) p_{i, j+1}+L_{\xi}^{\top}(1) p_{i j}\right) \\
& +4 \beta_{1}\left(L_{\xi \xi}^{\top}(-1) p_{i, j+1}-L_{\xi \xi}^{\top}(1) p_{i j}\right), \\
h \widehat{\partial_{x} \psi}= & \beta_{0}\left(L^{\top}(-1) \psi_{j+1}-L^{\top}(1) \psi_{j}\right)+L_{\xi}^{\top}(-1) \psi_{j+1}+L_{\xi}^{\top}(1) \psi_{j}, \\
\widehat{\psi}= & \frac{1}{2}\left(L^{\top}(1) \psi_{j}+L^{\top}(-1) \psi_{j+1}\right) .
\end{aligned}
$$

This when substituted into (2.12c) gives

$$
A \psi_{j-1}+B \psi_{j}+C \psi_{j+1}=h K \sum_{i=1}^{m} q_{i} c_{i j}+\frac{h^{2}}{2} \sum_{n=1}^{Q_{1}} \omega_{n} \rho_{0}\left(x_{j}+h s_{n} / 2\right) L\left(s_{n}\right),, \quad 2 \leq j \leq N-1,
$$

where

$$
\begin{aligned}
A= & -L(-1)\left(\beta_{0} L(1)-L_{\xi}(1)\right)^{\top}-L_{\xi}(-1) L^{\top}(1) \\
B= & 2 \int_{-1}^{1} L_{\xi} L_{\xi}^{\top} d \xi+L(-1)\left(\beta_{0} L(-1)+L_{\xi}(-1)\right)^{\top}+L(1)\left(\beta_{0} L(1)-L_{\xi}(1)\right)^{\top} \\
& +L_{\xi}(-1) L^{\top}(-1)-L_{\xi}(1) L^{\top}(1), \\
C= & -L(1)\left(\beta_{0} L(-1)+L_{\xi}(-1)\right)^{\top}+L_{\xi}(1) L^{\top}(-1), \\
K= & \frac{h}{2} \int_{-1}^{1} L(\xi) L^{\top}(\xi) d \xi
\end{aligned}
$$

Here $Q_{1}$-point Gauss quadrature rule on the interval $(-1,1)$ is used to integrate $\rho_{0}\left(x_{j}+h \xi / 2\right) L(\xi)$. We choose $Q_{1} \geq \frac{k+2}{2}$ points so that the quadrature rule has accuracy of at least $O\left(h^{k+2}\right)$ order. Boundary conditions are specified in section 2.2. Note that the resulting $(k+1) N \times(k+1) N$ matrix is a sparse block matrix, which can be inverted efficiently.

We then compute $(2.12 \mathrm{~b})$ by the same quadrature rule

$$
K p_{i j}=q_{i} K \psi_{j}+\frac{h}{2} \sum_{n=1}^{Q_{1}} \omega_{n} \log \left(L^{\top}\left(s_{n}\right) c_{i j}\right) L\left(s_{n}\right) .
$$

Finally we simplify (2.12a) to get the following ODE system

$$
K \dot{c}_{i j}=\frac{2}{h} R_{1}+\frac{1}{2 h}\left(R_{2}+R_{3}\right), \quad 2 \leq j \leq N-1,
$$


where

$$
\begin{aligned}
R_{1}= & -\sum_{n=1}^{Q_{2}} \omega_{n} L^{\top}\left(s_{n}\right) c_{i j} L_{\xi}^{\top}\left(s_{n}\right) p_{i j} L_{\xi}\left(s_{n}\right), \\
R_{2}= & \left(L^{\top}(1) c_{i j}+L^{\top}(-1) c_{i, j+1}\right)\left(-D^{\top} p_{i j}+E^{\top} p_{i, j+1}\right) L(1) \\
& \quad-\left(L^{\top}(1) c_{i, j-1}+L^{\top}(-1) c_{i j}\right)\left(-D^{\top} p_{i, j-1}+E^{\top} p_{i j}\right) L(-1)=R_{2}^{+}-R_{2}^{-}, \\
R_{3}= & \left(L^{\top}(1) c_{i j}+L^{\top}(-1) c_{i, j+1}\right)\left(L^{\top}(1) p_{i j}-L^{\top}(-1) p_{i, j+1}\right) L_{\xi}(1) \\
& \quad+\left(L^{\top}(1) c_{i, j-1}+L^{\top}(-1) c_{i j}\right)\left(L^{\top}(1) p_{i, j-1}-L^{\top}(-1) p_{i j}\right) L_{\xi}(-1)=: R_{3}^{+}+R_{3}^{-} .
\end{aligned}
$$

Here

$$
D=\beta_{0} L(1)-L_{\xi}(1)+4 \beta_{1} L_{\xi \xi}(1), \quad E=\beta_{0} L(-1)+L_{\xi}(-1)+4 \beta_{1} L_{\xi \xi}(-1) .
$$

In the evaluation of $R_{1}$, we choose $Q_{2} \geq \frac{k+4}{2}$ points. In implementation, since $Q_{2}>Q_{1}$, we simply use $Q_{2}$ Gaussian quadrature points in integrating both $\rho_{0}$ and $\log \left(c_{i}\right)$.

At two end cells, $R_{1}$ is still valid. The zero flux conditions (1.1d) are used to obtain $R_{2}$ and $R_{3}$, i.e., at $j=1$, we use $R_{2}=R_{2}^{+}, R_{3}=R_{3}^{+}$, and at $j=N$ we use $R_{2}=-R_{2}^{-}, R_{3}=-R_{3}^{-}$. The discrete ODE system is then solved by the RK2 method (3.43).

\section{REFERENCES}

[1] A. Arnold, P. Markowich and G. Toscani. On large time asymptotics for drift-diffusion-Poisson systems. Transport Theory and Statistical Physics, 29(3-5):571-58, 2000.

[2] A.M. Anile, N. Nikiforakis, V. Romano and G. Russo. Discretization of semiconductor device problems. II. Handbook of numerical analysis, vol. XIII. Handb. Numer. Anal. XIII, 443-522, 2005.

[3] M.Z. Bazant, K.T. Chu and B.J. Bayly. Current-voltage relations for electrochemical thin films. SIAM J. Appl. Math. 65:1463, 2005.

[4] M. Burger, V. Capasso and D. Morale. On an aggregation model with long and short range interactions. Nonlinear Analysis: Real World Applications, 8:939-958, 2007.

[5] A. Blanchet, J. A. Carrillo and P. Laurencot. Critical mass for a Patlak-Keller-Segel model with degenerate diffusion in higher dimensions. Calc. Var. Partial Differential Equations, 35(2):133-168, 2009.

[6] P. Biler and J. Dolbeault. Long time behavior of solutions to Nernst-Planck and Debye-Hückel drift-diffusion systems. Annales Henri Poincaré, 1(3):461-472, 2000.

[7] M. Burger and M. D. Francesco. Large time behavior of nonlocal aggregation models with nonlinear diffusion. Netw. Heterog. Media, 3(4):749-785, 2008.

[8] P. Biler, W. Hebisch and T. Nadzieja. The Debye system: existence and large time behavior of solutions. Nonlinear Anal., 23:1189-1209, 1994.

[9] F. Brezzi, L. Marini, S. Micheletti, P. Pietra, R. Sacco and S. Wang. Discretization of semiconductor device problems. I. Handbook of numerical analysis, vol. XIII. Handb. Numer. Anal. 13, 317-441, 2005.

[10] J. Bedrossian, N. Rodríguez and A. L. Bertozzi. Local and global well-posedness for aggregation equations and Patlak-Keller-Segel models with degenerate diffusion. Nonlinearity, 24:168-714, 2011.

[11] D.S. Bolintineanu, A. Sayyed-Ahmad, H.T. Davis and Y.N. Kaznessis. Poisson-Nernst-Planck models of nonequilibrium ion electrodiffusion through a protegrin transmembrane pore. PLoS Comput. Biol. 5(1):e1000277, 2009.

[12] H. Cohen and J. Cooley. The numerical solution of the time-dependent Nernst-Planck equations. Biophys. J. 5:145-162, 1965.

[13] A.E. Cardenas,R.D. Coalson and M.G. Kurnikova. Three-dimensional Poisson-Nernst-Planck theory studies: Influence of membrane electrostatics on gramicidin A channel conductance. Biophys. J. 79(1):80-93, 2000.

[14] C. Chainais-Hillairet and F. Filbet. Asymptotic behavior of a finite volume scheme for the transient driftdiffusion model. IMA J. Numer. Anal., 27(4):689-716, 2007.

[15] C. Chainais-Hillairet, J. G. Liu and Y. J. Peng. Finite volume scheme for multi-dimensional drift-diffusion equations and convergence analysis. M2AN Math. Model. Numer. Anal. 37(2):319-338, 2003.

[16] J.H. Chaudhry, J. Comer, A. Aksimentiev and L. Olson. A stabilized finite element method for modified PoissonNernst- Planck equations to determine ion flow through a nanopore. Commun Comput Phys., $15(1), 2014$.

[17] S. Datta. Electronic Transport in Mesoscopic Systems. Cambridge University Press, 1997. 
[18] B. Eisenberg and W. Liu. Poisson-Nernst-Planck systems for ion channels with permanent charges. SIAM J. Math. Anal. 38: 1932-1966, 2007.

[19] W. Fang and K. Ito. Global solutions of the time-dependent drift-diffusion semiconductor equations. J. Differential Equations, 123: 523-566, 1995.

[20] W. Fang and K. Ito. Asymptotic behavior of the drift-diffusion semiconductor equations. J. Differential Equations, 123: 567-587, 1995.

[21] H. Gajewski and K. Gröger. On the basic equations for carrier transport in semiconductors. J. Math. Anal. Appl., 113: 12-35, 1986.

[22] H. Gajewski and K. Gärtner. On the discretization of Van Roosbroeck's equations with magnetic field. Z. Angew. Math. Mech., 76(5):247-264, 1996.

[23] S. Glasstone. An introduction to Electrochemstry. D. Van Nostrand Company, Inc., Princeton, NJ, 1942.

[24] J. S. Hesthaven and T. Warburton. Nodal Discontinuous Galerkin Methods: Algorithms, Analysis, and Applications. Springer, New York, 2007.

[25] B. Hille. Ion channels and Excitable Membranes. 3rd ed. (Sinauer Associates, Inc., Sunderland, MA, 2001).

[26] J. W. Jerome. Analysis of Charge Transport: A Mathematical Study of Semiconductor Devices. Springer, Berlin, 1996.

[27] M.G. Kurnikova, R.D. Coalson, P. Graf and A. Nitzan. A lattice relaxation algorithm for three- dimensional Poisson-Nernst-Planck theory with application to ion transport through the gramicidin A channel. Biophys J. $76: 642-656,1999$.

[28] D. Li. Electrokinetics in Microfluidics. Vol. 2, Academic Press, 2004.

[29] H. Liu. Optimal error estimates of the direct discontinuous Galerkin method for convection-diffusion equations. Math. Comput., 84 (295): 2263-2295, 2015

[30] H. Liu and Z. Wang. A free energy satisfying finite difference method for Poisson-Nernst-Planck equations. J. Comput. Phys., 268:363-376, 2014.

[31] H. Liu and Z. Wang. Entropy satisfying discontinuous Galerkin methods nonlinear Fokker-Planck equations. J. Sci. Comput., in press, 2016.

[32] H. Liu and J. Yan. The direct discontinuous Galerkin (DDG) methods for diffusion problems. SIAM J. Numer. Anal., 47: 675-698, 2009.

[33] H. Liu and J. Yan. The direct discontinuous Galerkin (DDG) method for diffusion with interface corrections. Commun. Comput. Phys., 8(3):541-564, 2010.

[34] H. Liu and H. Yu. An entropy satisfying conservative method for the Fokker-Planck equation of the finitely extensible nonlinear elastic dumbbell model. SIAM J. Numer. Anal., 50:1207-1239, 2012.

[35] H. Liu and H. Yu. The entropy satisfying dicontinuous Galerkin method for Fokker-Planck equations. J. Sci. Comput. 62: 803-830, 2015.

[36] H. Liu and H. Yu. Maximum-Principle-Satisfying Third Order Discontinuous Galerkin Schemes for FokkerPlanck Equations. SIAM J. Sci. Comput., 36(5):A2296-A2325, 2014.

[37] B. Lu, Y.C. Zhou, G.A. Huber, S.D. Bond, M.J. Holst and J.A. McCammon. Electrodiffusion: A continuum modeling framework for biomolecular systems with realistic spatiotemporal resolution. J. Chem. Phys. 127:135102, 2007.

[38] B. Z. Lu, M. J. Holst, J. A. McCammon and Y. C. Zhou. Poisson-Nernst-Planck equations for simulating biomolecular diffusion-reaction processes I: Finite element solutions. J. Comput. Phys., 229(19):6979-6994, 2010.

[39] P.A. Markowitch. The Stationary Semiconductor Device Equations. Springer, Wien, 1986.

[40] M.S. Mock. Analysis of Mathematical Models of Semiconductor Devices. vol. 3, Boole Press, 1983.

[41] M. Mirzadeha and F. Giboua. A conservative discretization of the Poisson-Nernst-Planck equations on adaptive Cartesian grids. J. Comput. Phys., 274:633-653, 2014.

[42] P.A. Markowich, C.A. Ringhofer and C. Schmeiser. Semiconductor Equations. Springer-Verlag Inc., New York, 1990.

[43] M. S. Metti, J. Xu and C. Liu. Energetically stable discretizations for charge transport and electrokinetic models. J. Comput. Phys., 306:1-18, 2016.

[44] W. Nernst. Die elektromotorische wirksamkeit der ionen. Z. Phys. Chem. 4, 1889.

[45] M. Planck. Über die erregung von electricität und wärme in electrolyten. Annu. Phys. Chem. $39,1880$.

[46] A. Prohl and M. Schmuck. Convergent discretizations for the NernstPlanckPoisson system. Numer. Math. 111: 591-630, 2009.

[47] B. Rivière. Discontinuous Galerkin Methods for Solving Elliptic and Parabolic Equations: Theory and Implementation, SIAM, Philadelphia, 2008. 
[48] C.-W. Shu. Discontinuous Galerkin methods: general approach and stability. In Numerical solutions of partial differential equations, Adv. Courses Math. CRM Barcelona, pages 149-201. Birkhäuser, Basel, 2009.

[49] C.-W. Shu and S. Osher. Efficient implementation of essentially non-oscillatory shock-capturing schemes. J. Comput. Phys., 77: 439-471, 1988.

[50] T. Sokalski and A. Lewenstam. Application of Nernst-Planck and Poisson equations for interpretation of liquidjunction and membrane potentials in real-time and space domains. Electrochemistry Communications, 3(3):107$112,2001$.

[51] T. Sokalski, P. Lingenfelter and A. Lewenstam. Numerical solution of the coupled Nernst-Planck and Poisson equations for liquid junction and ion selective membrane potentials. J. Phys. Chem. B, 107(11):2443-2452, 2003.

[52] C. M. Topaz, A. L. Bertozzi and M. A. Lewis. A nonlocal continuum model for biological aggregation. Bull. Math. Bio., 68:1601-623, 2006.

[53] G.W. Wei, Q. Zheng, Z. Chen and K. Xia. Variational multiscale models for charge transport. SIAM Rev., 54:699-754, 2012.

[54] Q. Zheng, D. Chen and G.-W. Wei. Second-order Poisson-Nernst-Planck solver for ion channel transport. J Comput Phys., 230(13):5239-5262, 2011.

${ }^{\dagger}$ Iowa State University, Mathematics Department, Ames, IA 50011

E-mail address: hliu@iastate.edu

‡ Florida International University, Department of Mathematics and Statistics, Miami, FL 33199

E-mail address: zwang6@fiu.edu 